\title{
Mechanisms of Mitochondria-Neurofilament Interactions
}

\author{
0. I. Wagner, ${ }^{1}$ J. Lifshitz, ${ }^{2}$ P. A. Janmey, ${ }^{1}$ M. Linden, ${ }^{3}$ T. K. McIntosh, ${ }^{2}$ and J.-F. Leterrier ${ }^{3}$ \\ ${ }^{1}$ Institute for Medicine and Engineering, Department of Physiology, University of Pennsylvania, Philadelphia, Pennsylvania 19104-6383, ${ }^{2} \mathrm{Head}$ Injury \\ Center, Department of Neurosurgery, University of Pennsylvania, Philadelphia, Pennsylvania 19104-6316, and ${ }^{3}$ Unité Mixte de Recherche 6658 Centre \\ National de la Recherche Scientifique, Groupe de Biologie des Interactions Cellulaires, Poitiers University, 86022 Poitiers, France
}

\begin{abstract}
Mitochondria are localized to regions of the cell where ATP consumption is high and are dispersed according to changes in local energy needs. In addition to motion directed by molecular motors, mitochondrial distribution in neuronal cells appears to depend on the docking of mitochondria to microtubules and neurofilaments. We examined interactions between mitochondria and neurofilaments using fluorescence microscopy, dynamic light scattering, atomic force microscopy, and sedimentation assays. Mitochondria-neurofilament interactions depend on mitochondrial membrane potential, as revealed by staining with a membrane potential sensitive dye (JC-1) in the presence of substrates/ADP or uncouplers (valinomycin/carbonyl cyanide $p$-(trifluoromethoxy)phenylhydrazone) and are affected by the phosphorylation status of neurofilaments and neurofilament sidearms. Antibodies against the neurofilament heavy subunit disrupt binding between mitochondria and neurofilaments, and isolated neurofilament sidearms alone interact with mitochondria, suggesting that they mediate the interactions between the two structures. These data suggest that specific and regulated mitochondrial-neurofilament interactions occur in situ and may contribute to the dynamic distribution of these organelles within the cytoplasm of neurons.
\end{abstract}

Key words: neurofilament-phosphorylation; neurofilament-sidearms; axonal organelle motility; atomic force microscopy; JC-1; dynamic light scattering

\section{Introduction}

The spatial distribution of mitochondria is important for their biological function, and cytoskeletal changes that disrupt their distribution can cause cell death. Recent studies with transgenic mice lacking the intermediate filament (IF) desmin (Milner et al., 2000; Linden et al., 2001) implicate IF in positioning mitochondria at their appropriate sites. In several cell lines, mitochondrial distribution is coordinated by both microtubules (MTs) and IFs (Summerhayes et al., 1983). In neurons, axonal mitochondrial transport can occur along both MTs and actin filaments, whereas neuronal cells lacking MT or actin filaments [but still retaining neurofilaments (NFs)] exhibit no mitochondrial motility (Morris and Hollenbeck, 1995). Mitochondrial distribution in cells is suggested to occur by a coordinated effect of MTs and actin filaments (Couchman and Rees, 1982; Krendel et al., 1998) with a role for IFs in muscle (Stromer and Bendayan, 1990; Reipert et al., 1999) and non-muscle cells (Toh et al., 1980; Mose-Larsen et al., 1982; Almahbobi et al., 1993; Collier et al., 1993).

Regulation of organelle motility can be accounted for by several alternative or complementary mechanisms including the orchestration of motor activation (Sheetz, 1999), switching between plus and minus end-directed motors, and random or regulated static attachment to stationary cytoskeletal elements

\footnotetext{
Received May 21, 2003; revised July 25, 2003; accepted July 31, 2003.

This work was supported by a Deutscher Akademischer Austouschdienst-Fellowship (German Academic Exchange Service) and National Institutes of Health Grant R01 GM56707. We thank members of the Janmey lab, Dr. Helim Aranda-Espinoza, Jennifer Pastore, and Paramjeet Randhawa for careful readings of this manuscript.

Correspondence should be addressed to Dr. Oliver Wagner, University of Pennsylvania, Institute for Medicine and Engineering, 1010 Vagelos Research Laboratories, 3340 Smith Walk, Philadelphia, PA 19104-6383. E-mail: owagner@mail.med.upenn.edu.

Copyright $\odot 2003$ Society for Neuroscience $\quad 0270-6474 / 03 / 239046-13 \$ 15.00 / 0$
}

(Leterrier et al., 1994; Hollenbeck, 1996). In axons, motility of mitochondria, distributed to sites of high ATP demand (e.g., growth cones, synapses, and nodes of Ranvier), is altered by the apparent docking to NFs and MTs (Hollenbeck, 1996), consistent with electron microscopic studies revealing abundant mitochondria-NF and -MT interconnections (Hirokawa, 1982). In addition to frequent stops and starts, elastic recoil, and reversal of direction, axonal mitochondria spend a considerable amount of time stationary, although presumably bound to NFs and MTs (Martz et al., 1984; Forman et al., 1987; Morris and Hollenbeck, 1993; Ligon and Steward, 2000).

Although an association between mitochondria and NFs is supported by many studies (Hirokawa, 1982; Leterrier et al., 1991, 1994), the regulation of mitochondria-NF interactions is uncharacterized. In fibroblasts, mitochondrial distribution can be maintained by the IF network even after treatment with MT inhibitors. In contrast, treatment with a mitochondrial membrane potential inhibitor can redistribute mitochondria within the cell (Dudani et al., 1990). In yeast, which lacks IFs, mitochondrial position and movements depend on the actin cytoskeleton (Smith et al., 1995), and yeast mitochondria-actin interactions are ATP- but not membrane potential-sensitive (Lazzarino et al., 1994). In neurons, however, Overly et al. (1996) found a close relationship between metabolic activity and motility of mitochondria: highly active mitochondria in proximal dendrites were less motile compared with mitochondria with low metabolic activity in axons.

To evaluate the potential regulation of mitochondria interactions with NFs, we studied the binding of isolated brain mitochondria and purified NFs in vitro. Effects of modifying mitochondrial homeostasis, the phosphorylation state of the NF or 
cleaved NF domains, and competition of unlabeled NF and anti-NF antibodies provide evidence for the specificity and conditions of mitochondrial-NF interactions.

\section{Materials and Methods}

Purification, labeling, and dephosphorylation of NF and NF-sidearms. Neurofilaments were purified from bovine spinal cords (obtained from a local slaughterhouse) according to a procedure described previously (Leterrier et al., 1996). After removal of the dura, spinal cords were homogenized in 1 vol per wet weight of ice-cold reassembly buffer (RB) containing $0.1 \mathrm{~m}$ Mes-NaOH, pH 6.8, 1 mM $\mathrm{MgCl}_{2}, 1 \mathrm{~mm}$ EGTA, and $0.1 \mathrm{~mm}$ PMSF. The homogenate was centrifuged at $78,000 \times g$ for $1 \mathrm{hr}$ at $4^{\circ} \mathrm{C}$. The supernatant was brought to $4 \mathrm{M}$ glycerol and kept for $3 \mathrm{hr}$ at $4^{\circ} \mathrm{C}$. The NF-glycerol mixture was then centrifuged at $78,000 \times g$ for $1 \mathrm{hr}$ at $4^{\circ} \mathrm{C}$. The turbid-white NF pellet, contaminated with soluble proteins and membranes, was resuspended in RB buffer. To label filaments, a final concentration of $12 \mathrm{~mm}$ rhodamine B succinimide (kindly provided by Dr. Rolands Vegners, Latvian Institute of Organic Synthesis, Riga, Latvia) was added to the protein solution $\left(30 \mathrm{~min}\right.$ incubation at $\left.4^{\circ} \mathrm{C}\right)$. A protease inhibitor mixture, as described previously (Leterrier et al., 1996), was added to the NF solution after a gentle two-stroke homogenization step using a Teflon-glass homogenizer. The NFs were centrifuged over a discontinuous sucrose gradient ( 1.5 and $0.8 \mathrm{M}$ sucrose in $\mathrm{RB}$ ) at $100,000 \times g$ for $14 \mathrm{hr}$ at $4^{\circ} \mathrm{C}$ to remove contaminating proteins and membranes. The crystal-clear NF pellet was gently homogenized with two strokes in RB containing $0.8 \mathrm{~m}$ sucrose and protease inhibitors. The purified NF suspension was dialyzed for $24 \mathrm{hr}$ at $4^{\circ} \mathrm{C}$ in $\mathrm{RB}$ containing 0.8 M sucrose and $1 \mathrm{~mm}$ PMSF to remove NF-bound polypeptides (Gou et al., 1998). The purity of NF preparations was routinely checked by densitometry of Coomassie-stained gels after SDS-PAGE. For the preparations used in these studies, the NF triplet subunits [NF-heavy chain (NF-H), NF-medium chain (NF-M), and NF-light chain (NF-L)] accounted for 95\% of the total protein (see Results). Trace amounts of tightly associated proteins such as the dynein/dynactin complex (Shah et al., 2000) remain tenaciously bound to NFs during purification. The NF suspension was stored in aliquots at $-70^{\circ} \mathrm{C}$ after rapid freezing in liquid nitrogen.

Sidearms (SAs) were cleaved from the NF backbone using $26 \mu \mathrm{g}$ $\alpha$-chymotrypsin per $3 \mathrm{mg} / \mathrm{ml} \mathrm{NF}$ (incubation at $30^{\circ} \mathrm{C}$ for $1 \mathrm{hr}$ ) according to Julien and Mushynski (1983) and Chin et al. (1989). To obtain SAs devoid of chymotrypsin, $7.1 \mathrm{mg}$ of NF was incubated with $11.2 \mathrm{U}$ agarose-bound $\alpha$-chymotrypsin (Sigma, St. Louis, MO) for $1 \mathrm{hr}$ at $30^{\circ} \mathrm{C}$ under constant mixing, and 2.6 mM PMSF was added to stop the reaction. Solubilized SAs were recovered in the supernatant after centrifugation at $100,000 \times g$ for $1 \mathrm{hr}$ at $4^{\circ} \mathrm{C}$, whereas the NF backbone and other degradation products remained insoluble (Julien and Mushynski, 1983; Chin et al., 1989). The soluble fraction contains $\sim 90 \%$ NF-H sidearms [apparent molecular weight (MW) $160 \mathrm{kDa}$ ] determined by densitometry of Coomassie-stained gels and a smaller amount of NF-M sidearms (apparent MW $121 \mathrm{kDa}$ ) (Julien and Mushynski, 1983; Chin et al., 1989). Phosphorylated sidearms from the NF-H chain (ctSA-H) and control, phosphorylated sidearms from the NF-M chain (ctSA-M) were separated from each other by chromatography on DEAE cellulose in imidazole buffers according to Chin et al. (1989). The purified SA fractions were dialyzed $24 \mathrm{hr}$ at $4^{\circ} \mathrm{C}$ against $\mathrm{RB}$ and protease inhibitors before storage as aliquots at $-70^{\circ} \mathrm{C}$.

NF dephosphorylation was accomplished using $5 \mathrm{U} / \mathrm{ml}$ alkaline phosphatase (from either Escherichia coli or calf intestinal type VII, Sigma) per $3 \mathrm{mg} / \mathrm{ml} \mathrm{NF}$ dialyzed against RB for $48 \mathrm{hr}$ at room temperature according to Eyer and Leterrier (1988). Dephosphorylation of solubilized SA was done by incubating $50 \mu \mathrm{g}$ of SA $(90 \% \mathrm{SA}-\mathrm{H})$ in RB plus $1 \mathrm{~mm}$ PMSF for $4 \mathrm{~d}$ at $4^{\circ} \mathrm{C}$ under constant agitation together with $125 \mathrm{U}$ agarose-bound alkaline phosphatase (Sigma), washed previously with distilled water and $\mathrm{RB}$. The agarose-bound enzyme was removed by centrifugation for $5 \mathrm{~min}$ at $5000 \times g$. Both untreated native (phosphorylated) and dephosphorylated SA were further dialyzed against RB, $0.8 \mathrm{~m}$ sucrose, $1 \mathrm{~mm}$ PMSF, to a final concentration of $0.1 \mathrm{mg} / \mathrm{ml}$. Identical amounts $(2 \mu \mathrm{g})$ of the two samples, ctSAs and dpSAs, were analyzed on 7.5\% SDS-PAGE either stained with Coomassie blue or transferred onto immobilon membranes and stained with antibodies against either phosphorylated or dephosphorylated NF (dpNF)-H epitopes (see Results).

The high phosphorylation level of native NF-H (close to 50 phosphates per molecule) is reflected by an unusually low electrophoretic mobility in SDS-PAGE (apparent MW $200 \mathrm{kDa}$ for a molecule of $110 \mathrm{kDa}$ ). Dephosphorylation by alkaline phosphatase induces an incubation time-dependent increase in electrophoretic mobility toward a final apparent MW of $160 \mathrm{kDa}$ for molecules containing a remaining 4-10 phosphates per mole, which cannot be removed by further incubation (constitutive phosphorylation sites) (Julien and Mushynski, 1982; Carden et al., 1985; Hisanaga et al., 1993). Under the conditions used in the present preparations in which the lowest apparent MW of dpNF-Hs (dephosphorylated NF-Hs) were $160 \mathrm{kDa}$, we thus estimate the remaining phosphorylation level of dpNF-Hs and dpSA-Hs (dephosphorylated sidearms from the NF heavy chain) as being close to these published values; however, no previous study has reported the enzymatic dephosphorylation of solubilized SA-Hs.

Protein concentration was determined using the Lowry assay (Lowry et al., 1951), and SDS-PAGE was performed using 7.5\% separation gels according to the method of Laemmli (1970). Immunoblots were done after the method of Towbin et al. (1979) using anti-NF antibodies specific for phosphorylated or dephosphorylated NF-H and NF-M epitopes (SMI 31, SMI 32; Sternberger Monoclonals, Lutherville, MD).

Purification and fluorescence labeling of mitochondria. All animal experiments were approved by the Institutional Animal Care and Use Committee of the University of Pennsylvania and were in strict accordance with the NIH Guide for the Care and Use of Laboratory Animals. Mitochondria were isolated from rat-brain cortex (Sprague Dawley, weighing 350-400 gm) following the procedure used by Sims (1990) (method B). Animals were killed by decapitation, and brains were rapidly removed and placed in ice-cold isolation buffer $(0.32 \mathrm{~m}$ sucrose, 2 mM EGTA(K), $10 \mathrm{~mm}$ Tris- $\mathrm{HCl}, \mathrm{pH}$ 7.4). Dissected cortices were homogenized in a $12 \%$ solution of Percoll (in isolation buffer) using seven strokes of a $4 \mathrm{ml}$ Teflon-glass homogenizer. The homogenate was added onto a Percoll gradient (40 and 26\%) and centrifuged at $31,000 \times g$ for 8 min at $4^{\circ} \mathrm{C}$. The band located on top of the $40 \%$ Percoll is the mitochondrial fraction that was removed and stabilized with $0.5 \mathrm{mg} / \mathrm{ml}$ BSA after two washing steps by centrifugation $\left(17,000 \times g\right.$ for $14 \mathrm{~min}$ at $4^{\circ} \mathrm{C}$ and $7300 \times g$ for 5 $\min$ at $4^{\circ} \mathrm{C}$ ) in isolation buffer. The final pellet (a mixture of mitochondria from neuronal and glial origins) was resuspended in isolation buffer and protein was determined using the Bio-Rad DC protein assay (BioRad, Richmond, CA). The purity of mitochondria was assessed by measuring marker enzymes, by respiratory control ratio, and by transmission electron microscopic (TEM) imaging to evaluate the amount of intact mitochondria with a condensed matrix relative to other membranous components (mainly synaptosomes) as reported recently (Lifshitz et al., 2003). These measurements show that these preparations consist of $85 \%$ intact mitochondria (see Results). The respiratory control ratio of the mitochondrial preparation was determined to be $4.49 \pm 0.39$, which is in accordance with values published by Sims (1990).

Fluorescence imaging was accomplished by staining mitochondria $(0.15 \mathrm{mg} / \mathrm{ml})$ in respiration buffer $(10 \mathrm{~mm}$ TRIS, $5 \mathrm{~mm}$ Tris-phosphate, 0.05 mм EDTA(K), $100 \mathrm{~mm} \mathrm{KCl,} 75$ mм D-mannitol, 25 mм sucrose, $\mathrm{pH}$ 7.4) with the membrane potential sensitive dye JC-1 ( $1.8 \mu \mathrm{M})$ (Molecular Probes, Eugene, OR). JC-1 [5,5',6,6'-tetrachloro-1,1',3,3'-tetraethylbenzimidazolylcarbocyanine iodide, $\mathrm{CBIC2}(3)]$ is a lipophilic cationic dye that forms a potential- and concentration-dependent nematic phase, consisting of J-aggregates. JC-1 in its monomeric form exhibits a green color $(525 \mathrm{~nm})$ when excited at $490 \mathrm{~nm}$, whereas J-aggregates exhibit a red color $(590 \mathrm{~nm})$ when excited at $490 \mathrm{~nm}$. In the presence of substrates ( $5 \mathrm{~mm}$ malate and $5 \mathrm{~mm}$ glutamate buffered with $2 \mu \mathrm{M} \mathrm{KOH}$ ) and ADP $(0.1 \mathrm{~mm}), \mathrm{JC}-1$ uptake into the mitochondrial matrix is concomitantly followed by aggregation of JC-1 monomers. Mitochondria with low membrane potential, e.g., nonenergized (in the absence of substrates and $\mathrm{ADP}$ ) or uncoupled mitochondria (in the presence of substrates, ADP, 1 $\mu \mathrm{M}$ carbonyl cyanide $p$-(trifluoromethoxy)phenylhydrazone (FCCP), and 90 nM valinomycin), do not accumulate JC-1 (Smiley et al., 1991; Salvioli et al., 1997). JC-1-stained energized mitochondria exhibit a redorange color for membrane potential of $\sim 140-160 \mathrm{mV}$ and red fluorescence for mitochondria with membrane potential up to $190 \mathrm{mV}$ (Reers et 
al., 1995). For some experiments, we reduced the JC-1 concentration (Reers et al., 1991) to a final concentration of $0.5 \mu \mathrm{M}$ to diminish J-aggregates (which shifts the color from red or orange-red to yellow or yellow-green, respectively). After incubation with JC-1 (15 min at room temperature), mitochondria were washed to remove excess dye by a gentle centrifugation step $(3 \mathrm{~min}$ at $2000 \times g)$.

Transmission electron microscopy and dynamic light scattering of mitochondria. Mitochondrial ultrastructure was determined to assess purity and integrity (Hackenbrock, 1966), and size distribution of purified mitochondria was used to estimate the relative proportions of the population. The final mitochondrial pellet was fixed overnight with $2.5 \%$ glutaraldehyde in cacodylate buffer at $4^{\circ} \mathrm{C}$ and processed for routine thin-section ultrastructural analysis by the Biomedical Imaging Core Laboratory (Department of Pathology and Laboratory Medicine, University of Pennsylvania). Nonconsecutive and nonoverlapping images were randomly selected and captured at 24,000 $\times$ magnification. Intact mitochondria appear as structures with a continuous outer membrane, a distinct inner membrane, and a compacted matrix compartment (Halestrap, 1989). NF-mitochondria complexes were fixed by $24 \mathrm{hr}$ diffusion at $4^{\circ} \mathrm{C}$ without mechanical perturbation by carefully loading the same volume of $2 \%$ glutaraldehyde on the sample. Further processing of the fixed sample was performed as described previously (Leterrier et al., 1994).

To quantify mitochondrial size distributions, we analyzed mitochondrial populations in suspension by dynamic light scattering (DLS) (Dynals, Protein Solution Inc., Charlottesville, VA). The relative size distribution of the mitochondrial population can be obtained using DLS based on analysis of photon correlation spectroscopy (Glatter et al., 1991). Size distribution histograms represent $100 \%$ of the population with hydrodynamic radii signified by relative proportions of the total population. The hydrodynamic radii are calculated assuming that the particles are spheres, but the compact prolate ellipsoidal shape of mitochondria in isolation (Kristal and Dubinsky, 1997) would introduce only a negligible error in the effective size.

DLS was also used to determine mitochondria-NF interactions: NFs attached to mitochondria would increase the effective mitochondrial hydrodynamic radius by increasing both the particle size and viscous drag at the surface of the mitochondrion-NF complex.

Fluorescence and atomic force microscopy. Fluorescence imaging was performed using glass slides and cover glasses that were carefully cleaned with ethanol and dried. Labeled mitochondria (energized or discharged) were incubated with NFs or SAs in respiration buffer (100-200 $\mu$ l) for 15 min at room temperature before a sample volume of $40 \mu \mathrm{l}$ was transferred to the glass slide using a cut pipette tip to avoid mechanical damage to mitochondria and NFs. A cover glass $(0.15 \mathrm{~mm}$ thickness) was placed gently onto the sample, excess fluid was removed using a tissue, and the sides of the glass slide were sealed with silicone grease. Fluorescence microscopy was performed using a Leica DM IRBE microscope (Leica $\mathrm{GmbH}$, Wetzlar, Germany) with a $100 \times$ oil objective lens, and images were captured using a Hammamatsu C4712-95 CCD-camera (Hammamatsu Corporation, Bridgewater, NJ) connected to the microscope via an optical 1.6× magnification tube. Openlab software (v. 2.2.5, Improvision Inc., Quincy, Boston, MA) was used for image processing.

Ratio imaging was done using Adobe Photoshop 6.0 (Adobe Systems, San Jose, CA) to measure the mean green and red intensities of a selected area including a single mitochondrion or a small $(<5)$ aggregate of mitochondria. NFs projecting away from the mitochondria were also included in the measuring area. Statistics were analyzed using the Student's $t$ test (one tailed, two sample unequal variance) on the basis of 15-50 measured mitochondria from three to six independently repeated experiments. Significance is taken as ${ }^{\star} p<0.05$.

Ratio imaging was performed with energized mitochondria using decreased JC-1 concentrations, so that the amount of bound rhodamine B-labeled NFs or SAs (red fluorescence) on mitochondria (yellowish fluorescence) increases the red to green ratio. The number of NFs or SAs bound to mitochondria was counted by an unbiased observer to compare the amount of NFs and SAs attached on energized, nonenergized, or uncoupled mitochondria. Count data are presented as histograms showing how many times a mitochondrion reveals a given number of NFs or
SAs bound in a sampled population. For each condition, 15-50 mitochondria were evaluated from three to six independent experiments. If sidearms appear to be in an aggregate of a radius three times larger than a single red spot, comparable with those on the glass surface of Figure 5, $A$ and $B$, we counted it as " 1 ," and thus there may be an underestimate and the effect of membrane potential-mediated SA binding may be more significant. However, the underestimate was comparable for all groups (our goal was not to determine an absolute number of sidearms but the ratio between SAs attached to energized and discharged mitochondria complementary to our ratio imaging). In the case of NFs, the objective observer was only counting single NFs with a clear-cut shape at the highest resolution (the 600 dpi images were zoomed to a maximum extent). An aggregate of NF was counted as "1," whereas an aggregate never contains more than five NFs (we did not count globs). The resulting underestimate was comparable for all groups.

Atomic force microscopy (AFM) imaging was done with a Bioscope IIIa instrument (Digital Instruments, Santa Barbara, CA). Unstained mitochondria (energized or discharged) were incubated with unlabeled NFs in respiration buffer for $15 \mathrm{~min}$ at room temperature. The sample $(300 \mu \mathrm{l})$ was transferred to freshly cleaved mica double taped on a plastic dish. Imaging was performed in fluid tapping mode using digital silicon nitride probe-sharpened oxide-sharpened silicon nitride probes (Digital Instruments, Santa Barbara, CA) with a spring constant of $0.32 \mathrm{~N} / \mathrm{m}$ at a scanning frequency of $0.8-1.2 \mathrm{~Hz}$. Images were processed using the Nanoscope (R) IIIa software (v. 5.12, Digital Instruments), and the WSxM freeware (v. 3.0, Nanotec, Madrid, Spain) was used for height measurements, flattening, three-dimensional (3D) conversion, and inserting scale bars. The G-scanner was calibrated using a standard grid. All buffers were filtered using a sterile $0.22 \mu \mathrm{m}$ mesh-size filter.

Sedimentation assay of sidearm binding to mitochondria. Increasing concentrations $(0-10 \mu \mathrm{g} / \mathrm{ml})$ of ctSA-Hs were incubated with $2 \mathrm{mg} / \mathrm{ml}$ mitochondria in STE buffer (0.32 $\mathrm{m}$ sucrose, $10 \mathrm{~mm}$ TRIS, $1 \mathrm{~mm}$ EDTA, protease inhibitors) containing $5 \mathrm{~mm}$ succinate in the presence or the absence of $2 \mu \mathrm{M}$ FCCP. Mitochondria were then separated from unbound soluble proteins by centrifugation for $10 \mathrm{~min}$ at $15,000 \times g$ onto $0.5 \mathrm{~m}$ sucrose in STE. Pellets were resuspended in denaturation buffer, and identical samples $(0.125 \mathrm{mg}$ of mitochondria proteins) were analyzed by $7.5 \%$ SDS-PAGE and immunoblotting with SMI 31 antibodies. A similar assay for dpSA-Hs could not be done because mitochondrial proteins were detected by SMI 32. Similarly, the binding of intact NFs to mitochondria could not be assayed by sedimentation because NFs cosediment with mitochondria whether bound or not, likely as the result of self aggregation of NFs (data not shown).

All reagents were obtained from Sigma unless indicated otherwise.

On-line material. A video sequence (videol.mov; $3 \mathrm{sec}$ total, five frames per second, 24 bit) of the uppermost mitochondrion shown in the first panel of Figure $3 \mathrm{~A}$ with attached NFs undulating in a shear flow (shear stress is $2.4 \times 10^{-3} \mathrm{~N} / \mathrm{m}^{2}$ ) is available on-line at www. jneurosci.org (file size is $3 \mathrm{MB}$ ). The shear stress exerted by the fluid flow on the NF bound to the mitochondrion is estimated from the relation $\tau=\eta(d v / d L)$ where $\tau$ is the wall shear stress, $\eta$ is the fluid viscosity $(1.2 \mathrm{mPa} \times \mathrm{sec})$ and the velocity gradient $d v / d L$ is derived from the linear velocity of the particle visible in the movie $(v=1$ $\mu \mathrm{m} / \mathrm{sec})$ and its height above the surface $(L=0.5 \mu \mathrm{m})$ estimated from the fact that the particle is approximately as well focused as the top of the mitochondrion. With these values, the shear stress $\tau$ is estimated as $2.4 \mathrm{mPa}=2.4 \times 10^{-3} \mathrm{~N} / \mathrm{m}^{2}$.

\section{Results}

\section{Imaging, size distribution, and functional characterization of mitochondria}

Figure $1 A$ shows mitochondria visualized by AFM. Mitochondria appear ellipsoidal (mean axial ratio of $1.8 \pm 0.2$ ) with an average diameter of $600 \pm 250 \mathrm{~nm}$ (determined from 25 mitochondria from three different preparations). The majority of the mitochondria are single particles, and a portion $(<15 \%)$ of the population exists in aggregates with fewer than eight mitochondria per aggregate. DLS measurements reveal a mean mitochon- 
A
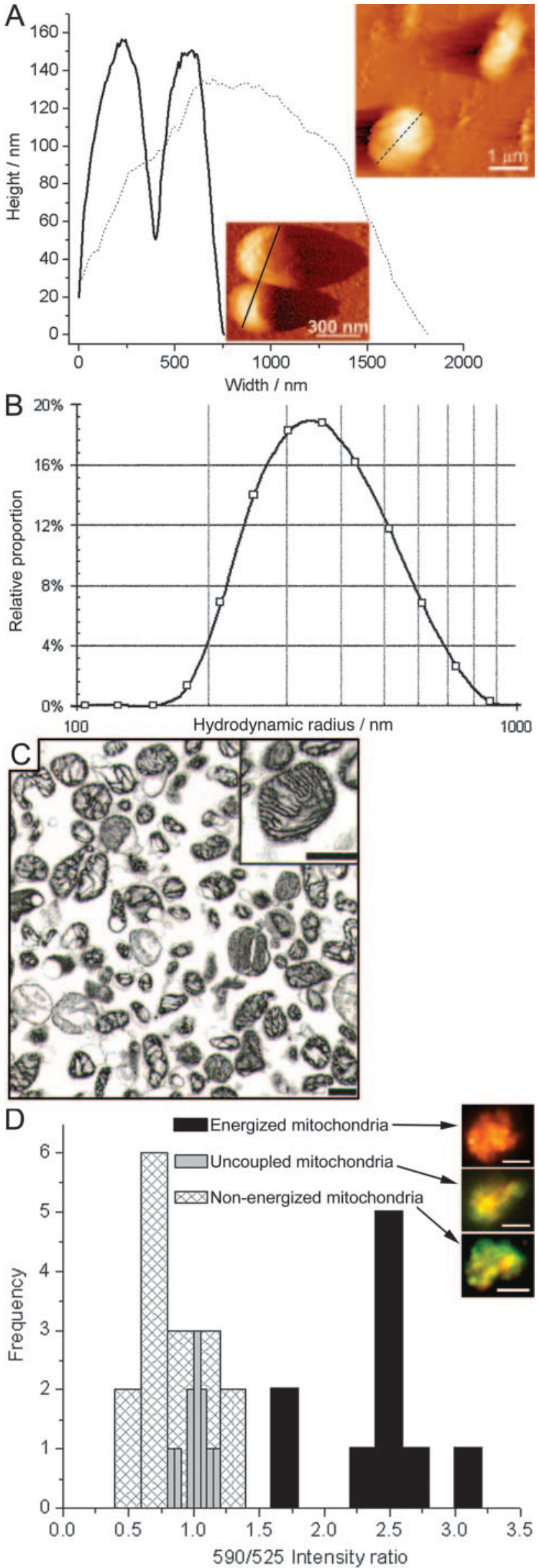

drial radius of $350 \mathrm{~nm}$ (Fig. 1B) in close agreement with AFM results. AFM height and width measurements reveal mitochondria as unexpectedly flattened (Fig. $1 A$, graph), presumably caused by adsorption to the mica surface. TEM imaging reveals $85 \%$ nondisrupted mitochondria with dense, intact matrices (Fig. 1C). The remaining membranous components are mainly synaptosomes (seen by TEM but not by AFM) and disrupted mitochondria.

To visualize the mitochondrial membrane potential, we stained mitochondria with JC-1, a lipophilic cationic dye that becomes concentrated inside mitochondria in proportion to the transmembrane potential. The least active mitochondria have relatively low transmembrane potentials, accumulate less dye, and appear green (nonenergized or uncoupled mitochondria). In contrast, the more active mitochondria (energized) have higher transmembrane potentials, leading to higher intramitochondrial dye concentrations and red fluorescence (Fig. 1D).

\section{Purification and imaging of phosphorylated and dephosphorylated NFs and sidearms}

$\mathrm{NFs}$, which are composed of three distinct subunits (NF-H, NF-M and NF-L) (Fig. 2A), appear in both fluorescence microscopy (Fig. $2 B$ ) and AFM (Fig. 2C) as flexible filaments when bound to a surface. NFs were extensively dephosphorylated by treatment with alkaline phosphatase (see Material and Methods) to investigate interactions between mitochondria and dpNFs (see below). Efficient dephosphorylation is revealed by an increased migration speed of the NF-H and NF-M bands in the SDSpolyacrylamide gel (Fig. $2 \mathrm{~A}$, second lane). The shift in electrophoretic mobility follows the time course of the alkaline phosphatase treatment, and the number of remaining phosphates is variable in different studies, depending on $\mathrm{pH}$, but does not exceed 10 per NF-H under the conditions used here ( $48 \mathrm{hr}$ ) (Julien and Mushynski, 1982; Carden et al., 1985; Hisanaga et al., 1993).

To investigate interactions between mitochondria and SAs shown previously to occur in vitro (Leterrier et al., 1991, 1994), we isolated and visualized phosphorylated and dephosphorylated SA obtained from the NF-H and NF-M subunits. In SDS-PAGE stained with Coomassie brilliant blue, the phosphorylated sidearms from the NF-H chain migrate as a single band (apparent MW $160 \mathrm{kDa}$ ), whereas the dephosphorylated sidearms from the NF-H chain appear in various configurations (migrating between apparent MW of 160 and $80 \mathrm{kDa}$ ), as detected by Western blots using monoclonal antibodies specific to phosphorylated (SMI 31 ) and dephosphorylated (SMI 32) NFs, respectively (Fig. 2D). The dephosphorylated SA-Hs were poorly stained by Coomassie blue, and a wide range of electrophoretic migration was revealed by immunoblotting with an antibody specific to NF-H. The un-

\footnotetext{
$\leftarrow$

Figure 1. Visualizing mitochondria with the AFM, fluorescence microscope, TEM, and DLS.A, AFM imaging (line scan revealed from the height image) bears mostly spherical-shaped mitochondria with diameters between $200 \mathrm{~nm}$ (solid line, bottom image) and $2 \mu \mathrm{m}$ (dotted line, top image), in agreement with DLS measurements ( $B$ ). C, TEM images (scale bar, $500 \mathrm{~nm}$ ) of ultrathin sections of isolated mitochondria showing intact (condensed) matrices, of a size distribution comparable with DLS measurements (B).D, Mitochondria $(0.15 \mathrm{mg} / \mathrm{ml})$ stained with 1.8 $\mu \mathrm{MJC}-1$. Energized mitochondria were treated with $5 \mathrm{~mm}$ malate, $5 \mathrm{~mm}$ glutamate, and $0.1 \mathrm{~mm}$ ADP. To uncouple mitochondria $1 \mu \mathrm{m} \mathrm{FCCP}$ and $90 \mathrm{~nm}$ valinomycin were added to energized mitochondria. The graph shows reproducible distributions of the ratio values of red-to-green fluorescence of $\mathrm{JC}-1$-stained mitochondria. For the fluorescence images of representative mitochondria for each condition, scale bar $=1 \mu \mathrm{m}$. Because some mitochondria are smaller than the wavelength of the emitted light (compare $A$ and $B, C$ ), they may appear larger in fluorescence images than they actually are.
} 


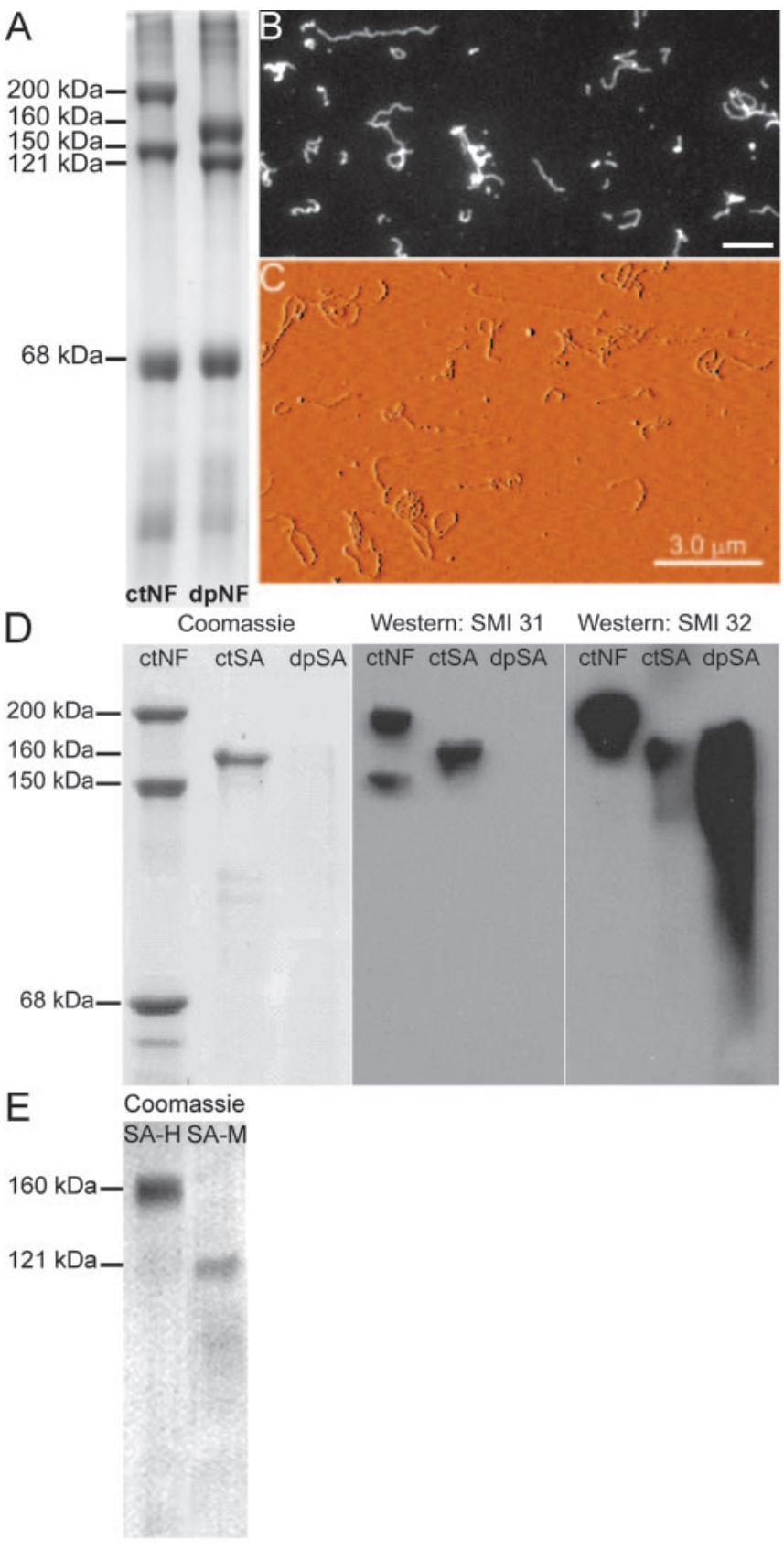

Figure 2. Visualization of NFs. A, SDS-PAGE and Coomassie brilliant blue staining of ctNFs (first lane) and dpNFs (second lane) (20 $\mu$ gloaded). The three NF subunits are named according to their migration speed in the gel and apparent MW (NF-H $=200 \mathrm{kDa}, \mathrm{NF}-\mathrm{M}=150 \mathrm{kDa}$, $\mathrm{NF}-\mathrm{L}=68 \mathrm{kDa}$ ). After extensive dephosphorylation (see Materials and Methods), the migration speed of the NF-H and the NF-M subunits increases as a result of phosphorylation-dependent conformational changes of their C-terminal domains (apparent MW 160 and $121 \mathrm{kDa}$, respectively). B, Fluorescence image of rhodamine B-stained NF (15 nM). C, AFM imaging of unstained $\mathrm{NF}$ (15 nм). No morphological difference between stained $(B)$ and unstained ( $C$ NFs was observed. $D$, After incubation of an NF suspension with $\alpha$-chymotrypsin followed by sedimentation of insoluble NFs (see Material and Methods), the supernatant contains mainly the C-terminal fragment of NF-H sidearms (ctSA-H, apparent MW $160 \mathrm{kDa}$ ) and trace amounts of NF-M (ctSA, first panel, $2 \mu \mathrm{g}$ loaded). After extensive dephosphorylation of the solubilized SAs with agarose-bound alkaline phosphatase, the Coomassie brilliant blue staining of SAs was nearly abolished (dpSA, first panel, $2 \mu \mathrm{g}$ loaded); however, immunoblotting of the same NF (10 $\mu \mathrm{g}$ loaded), ctSA, and dpSA fractions ( $2 \mu \mathrm{g}$ loaded) with antibodies specific to the phosphorylated (SMI 31, second panel) and dephosphorylated (SMI 32, third panel) NF-H/NF-M chains revealed the distinct migration pattern of $\mathrm{dpSA}-\mathrm{H}$ (third panel, third lane) compared with ctSA-H (second panel, second lane). E, SDS-PAGE and Coomassie brilliant blue staining of SAs after separation according to the method of Chin et al. (1989). Scale bar for the fluorescence image $=1 \mu \mathrm{m}$ usually wide range of electrophoretic mobilities produced after dephosphorylating this unique polypeptide (containing $\sim 50$ $\mathrm{KSP}$ repeats in the central domain of the sequence) is likely a consequence of conformational changes of the polypeptide induced by random sequential dephosphorylation, and not proteolysis, because all immunopositive bands migrate slower than predicted for a polypeptide with the sequence of the SA-H polypeptide $(\sim 62 \mathrm{kDa})$. Direct analysis of the same dpSA-H preparation by DLS and AFM revealed that the dpSA-H is intact and of the same extended size as the phosphorylated SA-H (Aranda-Espinoza et al., 2002). The small amount of NF-M sidearms (ctSA-M) (two polypeptides of apparent MW close to 121 $\mathrm{kDa}$ ) (Fig. $2 E$ ), is not detected by the two antibodies. In fluorescence micrographs, rhodamine-labeled ctSA-H and ctSA-M appear as diffuse spots with no evidence of remaining filamentous structures (data not shown).

\section{Phosphorylated NFs bind selectively to mitochondria with high membrane potential}

Rhodamine B-labeled NFs bind to energized JC-1-stained mitochondria (Fig. $3 A$, videol.mov), whereas most of the nonenergized (Fig. $3 B$ ) or uncoupled (Fig. 3C) mitochondria remain devoid of NF. Previous reports of fluorescence imaging of NFs in solution show that these filaments, unlike microtubules or F-actin, are so flexible that thermal motions cause them to coil over on each other with a length scale smaller than that resolved by light microscopy. When they are extended on a surface, NFs are straighter and obviously filamentous (Figs. $2 \mathrm{~B}, \mathrm{C}$ ) (and all the large fluorescence images showing a part of the glass surface), but when in solution (video1.mov) or on soft surfaces (e.g., bound to mitochondria) (Fig. 3A), they appear diffuse and wispy, as expected for such flexible polymers (Kas et al., 1996).

To improve visualization of rhodamine-labeled NFs (rhoNFs) on energized mitochondria, we decreased the JC-1 concentration (Reers et al., 1991) from 1.8 to $0.5 \mu \mathrm{M}$ (for $0.15 \mathrm{mg} / \mathrm{ml}$ mitochondria, $\mathrm{pH} 7.4$ ) to obtain a more yellowish color instead of red (compare Fig. $3 A$ with top image in Fig. $1 D$ ) and thereby reduce the contribution of JC-1 to the overall red fluorescence. In some cases we focused on the glass surface to estimate the amount of NFs in the sample (most of the larger images in this study) (Fig. $3 A$, second and third panels) and in some cases on top of the mitochondria away from the glass surface to ensure that binding occurs all around the mitochondria (the smaller images in Fig. 3 including only mitochondria) (Fig. $3 A$, first panel). Experiments were generally performed in sealed chambers, but in some cases we also examined NFs bound to energized mitochondria in shear flows, providing shear stresses of up to $2.4 \times 10^{-3} \mathrm{~N} / \mathrm{m}^{2}$ (see Material and Methods) to confirm that NFs were tightly bound and not simply overlying the mitochondrial surface (video1.mov shows the uppermost mitochondrion in the first panel of Fig. $3 \mathrm{~A}$ with attached NFs undulating in a shear flow). Adding FCCP (1 $\mu \mathrm{M})$ and valinomycin (90 nM) directly to an energized mitochondrion surrounded by NFs releases the filaments with concomitant discharging of the mitochondrial membrane (Fig. 3D).

To obtain higher resolution images in suspension and to confirm that the binding seen by fluorescence did not depend on the fluorophores, we also used AFM to visualize unstained mitochondria and unlabeled NFs. Before any AFM experiment was performed, a portion of freshly isolated mitochondria was used to detect the metabolic activity by JC-1 staining analogous to Figure $1 D$. The AFM results confirm that NFs frequently interact with energized mitochondria (Fig. $3 E, F$ ) but rarely with uncoupled 

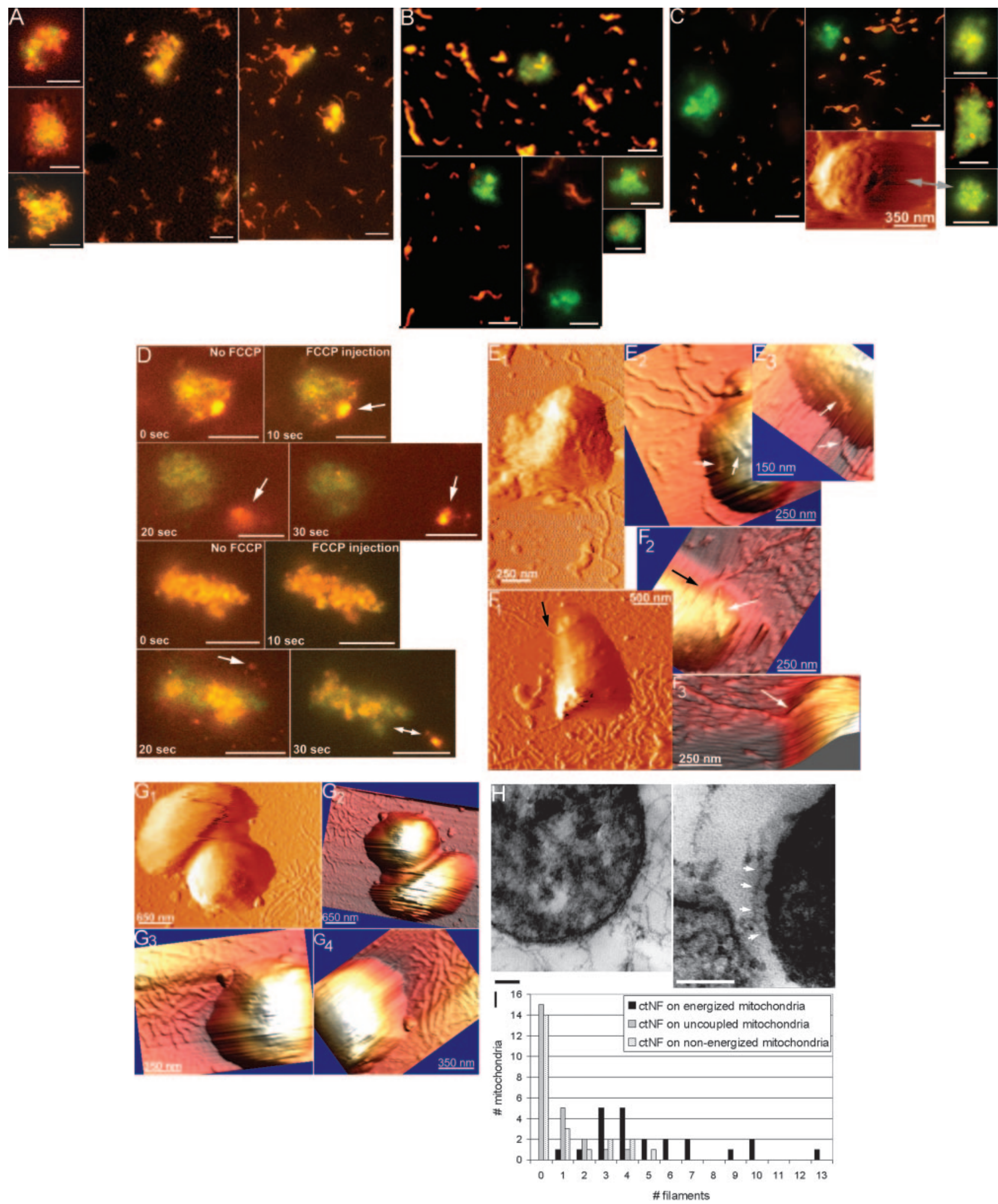

Figure 3. Interactions between ctNFs and mitochondria. $A$, Energized mitochondria $(0.15 \mathrm{mg} / \mathrm{ml})$ stained with reduced JC- 1 concentration $(0.5 \mu \mathrm{M})$ reveal a yellowish color (compare with Fig. $1 D$, top fluorescence image), allowing for visualization of both high membrane potential and bound rhodamine-labeled NFs ( $15 \mathrm{~nm})$, shown in pseudocolored red. The focus was both on the top of the mitochondria (small images, first panel) and near the glass surface (larger images, second and third panels). Nonenergized $(B)$ and uncoupled ( $C$ ) mitochondria do not bind to rho-NFs. Irregular surfaces of mitochondria were observed frequently in both AFM and fluorescence microscopy (AFM image in C). D, Two examples of online additions of FCCP (1 $\mu \mathrm{M})$ and valinomycin (90 nM) to mitochondria, which induced the release of bound NFs (white arrows) and a simultaneous discharge of mitochondrial membrane within seconds. $E_{1} F_{1}$ AFM amplitude $\left(E_{1}\right.$ and $\left.F_{7}\right)$ images (followed by $3 \mathrm{D}$ converted height images of different viewing angles, $E_{2,3}$ and $\left.F_{2,3}\right)$ of unstained (non-JC- 1 treated) energized mitochondria $(0.15 \mathrm{mg} / \mathrm{ml})$ bound to unlabeled NFs (15 $\mathrm{nM}$ ). The arrows point to NFs bound to the mitochondrial surface. $G$, Uncoupled mitochondria visualized by AFM in the presence of NFs reveal a smooth surface depleted of bound NFs $\left(G_{1}=\right.$ amplitude image; $G_{2-4}=3 D$ converted height image). $H$, TEM image of a mixture of isolated (energized) mitochondria and NFs showing that NFs bind the membrane en passant, through lateral interactions likely mediated by NF sidearms. Scale bar, $100 \mathrm{~nm}$. I, Quantification of ctNFs attached to energized, nonenergized, and uncoupled mitochondria (15-50 mitochondria from 2-6 independently repeated experiments), as revealed by fluorescence microscopy, counted by an unbiased observer. Scale bar for the fluorescence images of representative mitochondria for each condition $=1 \mu \mathrm{m}$. 
Table 1. Mean numbers of control (phosphorylated) filaments (ctNFs) and sidearms (ctSAs) binding per mitochondrion (data from Figs. 3l, 5F)

\begin{tabular}{lll}
\hline & ctNFs & ctSAs \\
\hline Energized & $5.3 \mathrm{NF} /$ mito & $20 \mathrm{SA} /$ mito \\
Nonenergized & $1.0 \mathrm{NF} /$ mito $(p<0.01)$ & $7.7 \mathrm{SA} / \mathrm{mito}(p<0.01)$ \\
FCCP & $0.7 \mathrm{NF} /$ mito $(p<0.01)$ & $5.6 \mathrm{SA} / \mathrm{mito}(p<0.01)$ \\
\hline
\end{tabular}

Statistics were analyzed using Student's $t$ test on the basis of the comparison of energized states with the nonenergized or uncoupled states. mito, Mitochondrion.

mitochondria (Fig. 3G; note the overall smoother mitochondrial surface compared with $E$ and $F$ ).

To reveal higher resolution images of energized mitochondria interacting with NFs on fixed samples, however, we used TEM showing NFs bound to the mitochondrial membrane en passant through lateral interactions likely mediated by NF sidearms (Fig. $3 H$ ).

To quantify mitochondria-NF interactions revealed from fluorescence imaging, the number of NFs bound to mitochondria under energized, nonenergized, or uncoupled conditions was counted by an observer unaware of the specific experimental conditions. Under the conditions of the incubation $(0.15 \mathrm{mg} / \mathrm{ml} \mathrm{mi-}$ tochondria and $15 \mathrm{nM} \mathrm{NF})$, an average of $5.3(n=116)$ neurofilament fragments were bound per mitochondrion in the energized state compared with $1.0(n=24)$ and $0.7(n=16)$ in the nonenergized and uncoupled states, respectively (Fig. 3I, Table 1).

\section{Mitochondria-NF interactions as revealed by DLS}

To verify that the membrane potential-mediated mitochondria-NF associations observed by microscopy can also be documented in equilibrium by a different experimental approach, we used photon correlation spectroscopy to follow changes in hydrodynamic radii on interactions of mitochondria with NFs. The contribution of NFs alone to the average hydrodynamic radius is very low (Fig. 4A, last two bars), and a simple additive effect would lower the average diameter compared with mitochondria alone, whereas the hydrodynamic radius of the mixture increases with NFs bound to mitochondria. The addition of NFs ( $30 \mathrm{nM}$ ) to energized mitochondria $(0.1 \mathrm{mg} / \mathrm{ml})$ increases the hydrodynamic radius of energized mitochondria, whereas the addition of $1 \mu \mathrm{M}$ FCCP and $90 \mathrm{~nm}$ valinomycin (to the same cuvette of a mitochondria-NF mixture) immediately decreases the mitochondrial hydrodynamic radius almost to the value of mitochondria alone (Fig. $4 A$, third and fourth bar). Figure $4 B$ shows a size distribution histogram representing the relative proportion (of $100 \%$ of the total population) (compare Fig. $1 B$ ) of hydrodynamic radii for the mitochondria-NF experiments shown in Figure $4 \mathrm{~A}$.

\section{Phosphorylated NF-sidearms bind selectively to mitochondria with high membrane potential}

To examine whether the mitochondria-NF binding is mediated by ctSAs (Hirokawa, 1982; Leterrier et al., 1991, 1994), we repeated fluorescence imaging using only the isolated labeled SA in place of the whole NF. Rhodamine B-stained ctSA-Hs bind in a concentration-dependent manner to mitochondria [Fig. 5, compare $A(0.09 \mu \mathrm{g} / \mathrm{ml} \mathrm{ctSA}), B(0.36 \mu \mathrm{g} / \mathrm{ml} \mathrm{ctSA})]$. Binding of ctSA-H is strongly reduced in nonenergized (Fig. $5 \mathrm{C}$ ) or uncoupled (Figs. 5D) mitochondria.

The fluorescence imaging evidence was confirmed using a sedimentation assay showing reduced binding of ctSA-Hs to uncoupled mitochondria compared with binding to energized mitochondria (Fig. 5E).
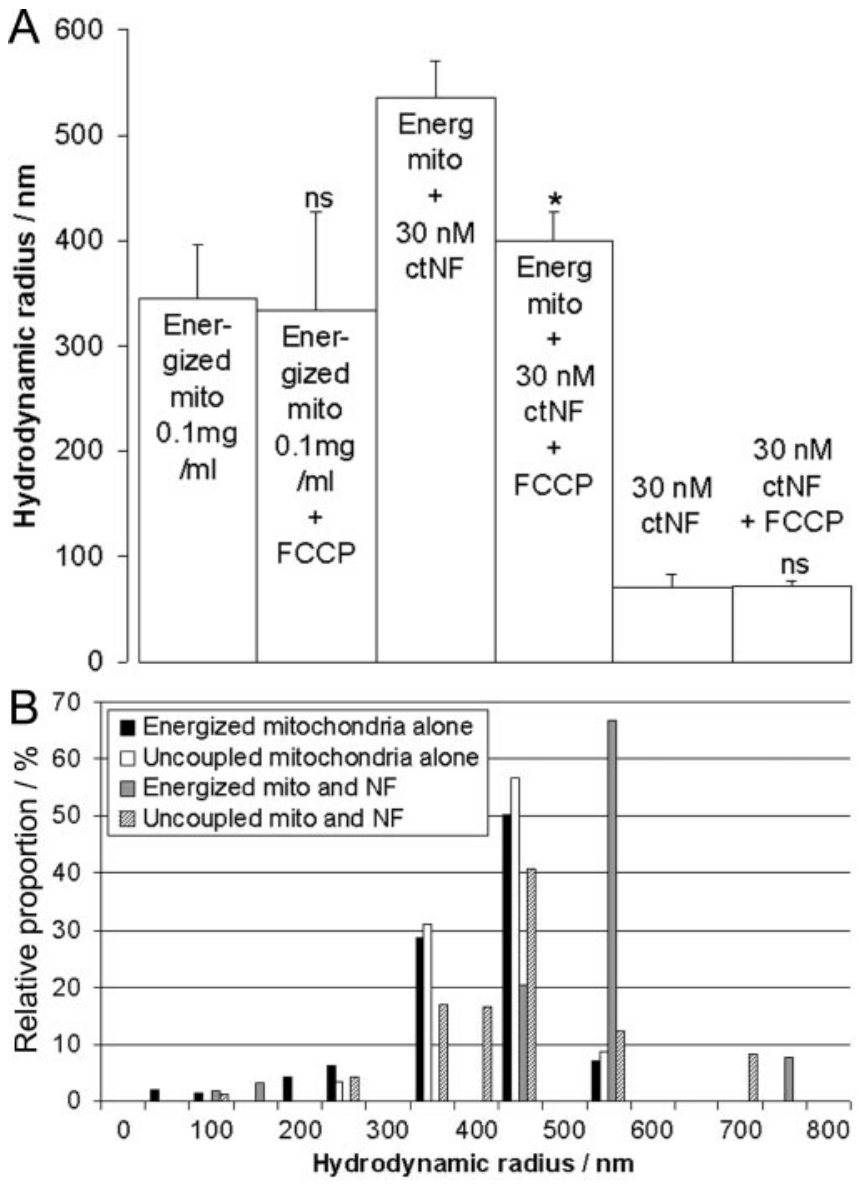

Figure 4. Mitochondrial hydrodynamic radii after reversible interactions with NFs in equilibrium. $A$, Hydrodynamic radius of energized and uncoupled mitochondria $(0.1 \mathrm{mg} / \mathrm{ml})$ with and without NFs ( $30 \mathrm{~nm}$ ) revealed by DLS. Unlabeled NFs added to unstained mitochondria induced a consecutive increase of the hydrodynamic radius of energized mitochondria (third bar). The addition of FCCP (1 $\mu \mathrm{M})$ and valinomycin ( $90 \mathrm{nM})$ to the same cuvette of a mitochondria-NF mixture determined the reduction of the radius close to the value of mitochondria alone (compare second and fourth bar). The contribution of the hydrodynamic radius of NFs alone is negligible (last two bars). $B$, Size distribution histogram representing the relative proportion (of $100 \%$ of the total population) (compare Fig. $1 B$ ) of hydrodynamic radii for the mitochondria-NF experiments shown in $A$.

Data from fluorescence imaging were quantified by counting single red dots (single or aggregated sidearms) on the mitochondrial surface (Fig. $5 F$ ). This analysis revealed a preferential binding of ctSA-Hs to energized mitochondria similar to that observed for intact control, phosphorylated neurofilaments (ctNFs): an average of $20(n=240)$ dots containing ctSA-Hs were bound per mitochondrion in the energized state compared with $7.7(n=69)$ and $5.6(n=39)$ in the nonenergized or uncoupled states, respectively (Fig. 5F, Table 1).

\section{Specificity of mitochondria-NF interactions}

To investigate the specificity of interactions between energized mitochondria and ctNFs, we first preincubated mitochondria with 90, 15, 3, or $0 \mathrm{~nm}$ unlabeled NFs for $20 \mathrm{~min}$ before adding 15 nM rho-NFs. Figure $6 \mathrm{~A}$ (open bars) shows the concentrationdependent increase in red fluorescence caused by increasing binding of rho-NFs with decreasing amounts of unlabeled NFs.

To further investigate the specificity of mitochondria-NF interactions, ctNFs were incubated with monoclonal anti-NF antibodies specific to ctNF epitopes. Ratio imaging (the red to green ratio increases after binding of rho-NFs to mitochondria) shows 

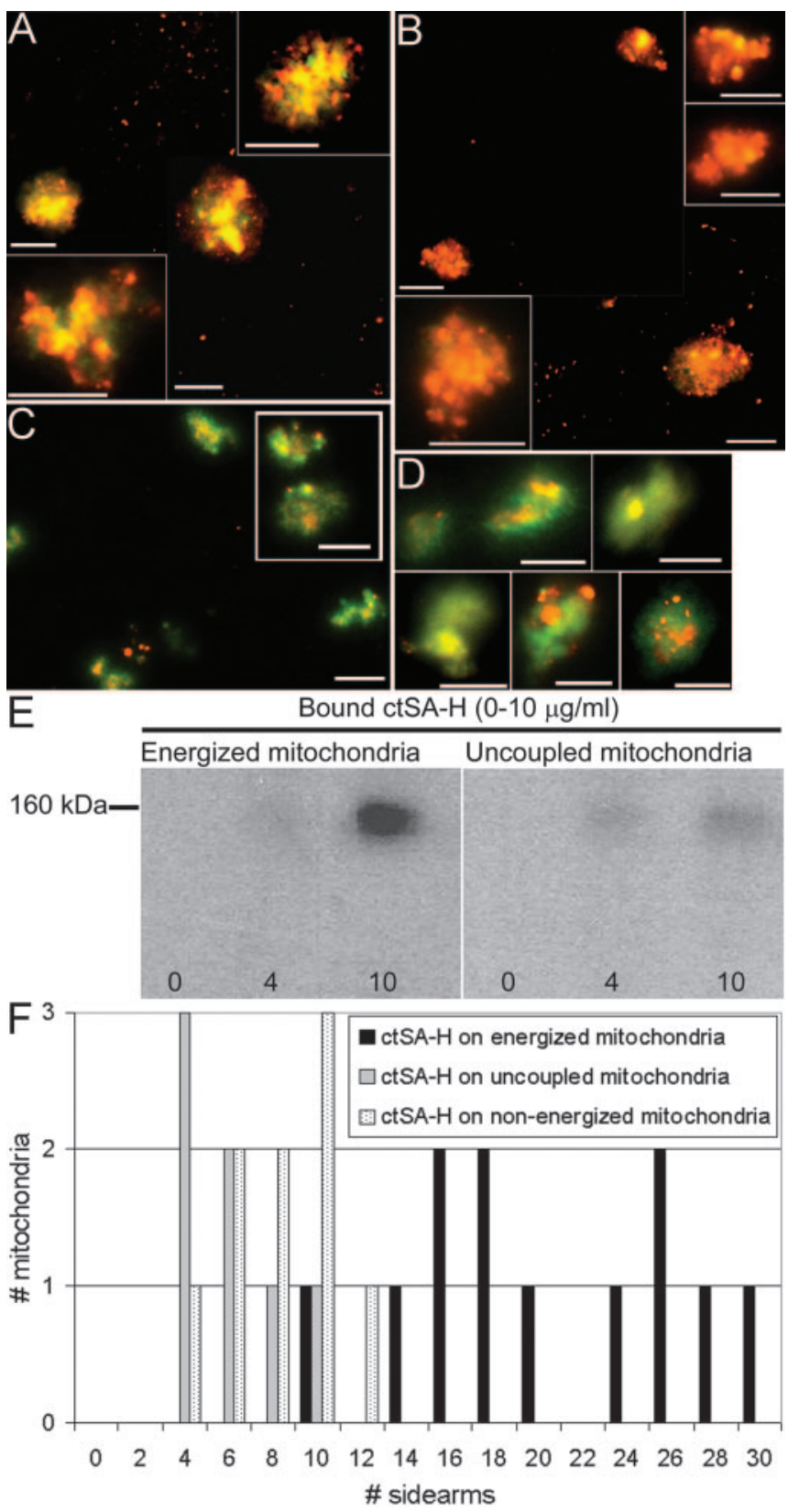

Figure 5. Phosphorylated NF-sidearms bind to energized mitochondria. $A, B$, Binding of rhodamine-labeled ctSA-Hs at low $(0.09 \mu \mathrm{g} / \mathrm{ml})(A)$ and high $(0.36 \mu \mathrm{g} / \mathrm{ml})(B)$ concentrations to energized mitochondria in the presence of ADP and substrates. The ctSA-Hs appear as small (pseudocolored) red dots on the mitochondrial surface. $C, D$, Nonenergized $(C)$ and uncoupled (D) mitochondria lack binding ctSA-Hs $(0.36 \mu \mathrm{g} / \mathrm{ml})$. E, Binding of increasing concentrations $(0-10 \mu \mathrm{g} / \mathrm{ml})$ of ctSA-Hs incubated with $2 \mathrm{mg} / \mathrm{ml}$ mitochondria followed by separation of mitochondria by sedimentation (see Materials and Methods). Western blotting using the monoclonal anti-NF antibody (SMI31) shows that mitochondria-bound ctSA-Hs were obviously reduced after discharging the mitochondria membranes with FCCP. F, Quantification of rhoctSA-H $(0.1 \mu \mathrm{g} / \mathrm{ml})$ binding to mitochondria reveals a similar binding pattern as observed for the whole filament (compare Fig. 3/). Scale bar of representative mitochondria for each condition $=1 \mu \mathrm{m}$

that the binding of NFs to mitochondria is significantly reduced by the addition of anti-NF antibodies (Fig. $6 \mathrm{~A}$, closed bars). The ratio imaging evidence of the specificity of ctNF binding to mitochondria was confirmed by the counting method, which shows a similar trend (Fig. 6 B, C). Binding of both ctSA-Hs (open bars) and ctSA-Ms (closed bars) to mitochondria was also inhibited by the anti-NF antibody SMI 31 (Fig. 6D). SAs from the medium chain also bound energized mitochondria, although the binding is statistically lower $(p<0.01)$ compared with that of the heavy chain (Fig. 6D, compare first and fourth bar). Fluorescence imaging revealed no binding of ctSA-M to uncoupled mitochondria (data not shown). The ratio imaging evidence of the specificity of sidearm binding to mitochondria was confirmed by the counting method, which shows a similar trend (Fig. 6E,F).

\section{Neurofilament dephosphorylation affects binding to discharged mitochondria}

In vitro dpNFs bind energized (Fig. 7A) mitochondria, but unlike ctNFs (Fig. 3, Table 1), they also appear to bind nonenergized (Fig. $7 B$ ) and uncoupled mitochondria (Fig. 7C). An average of $6.3(n=187)$ dpNF fragments were bound per mitochondrion in the energized state compared with $5.4(n=75)$ and $2.2(n=92)$ in the nonenergized or uncoupled states, respectively (Fig. $7 D$, Table 2). The binding specificity of dpNFs to mitochondria was assessed using the monoclonal anti-NF antibody SMI 32, specific to dephosphorylated epitopes (Fig. 2D, third panel). Binding of dpNFs to mitochondria was significantly decreased in the presence of the antibody (Fig. 7D, shaded bars). Specificity of binding of unlabeled ctNFs and dpNFs to unstained mitochondria was further confirmed using DLS (Fig. 7E).

Fluorescence imaging reveals that binding of dpSA-Hs to energized mitochondria is reduced compared with ctSA-Hs (data not shown). As observed for the dpNFs, there is still binding of dpSA-Hs to nonenergized mitochondria, whereas binding to uncoupled mitochondria is diminished (data not shown). Quantification of the data show binding of an average of $2.7(n=32)$ dpSA-Hs to energized, $3.3(n=59)$ to nonenergized, and 1.8 ( $n=$ 21 ) to uncoupled mitochondria (Fig. 8, Table 2). Table 2 shows that the mean numbers of $\mathrm{dpNF}$ and dpSA-H binding to mitochondria are close for energized and nonenergized mitochondria, whereas they are smallest for FCCP-treated mitochondria.

\section{Discussion}

Mitochondrial motility and metabolic function are coordinated: mitochondria are transported to regions where ATP consumption is high, and they disperse when the ATP/ADP ratio increases (Fawcett, 1981; Kellermayer et al., 1986; Aw and Jones, 1988; Wong-Riley, 1989; Smiley et al., 1991; Morris and Hollenbeck, 1993; Bereiter-Hahn and Vöth, 1994; Overly et al., 1996). Conversely, the nonrandom accumulation of mitochondria at certain sites may establish gradients of metabolites used or produced by mitochondria. The association of mitochondria at energyconsuming sites suggests, in addition to active movement along actin or MTs, the requirement for regulated immobilization at these sites. Net velocity and displacement of mitochondria in axons depends on the relative contribution of stationary and motile events. In neuronal processes, approximately one-third of the mitochondria are in motion at any time, whereas the remainder are stationary (Forman et al., 1987; Morris and Hollenbeck, 1995), and ultrastructural studies show cross-bridges from mitochondria to NFs (Fig. 3H) (Hirokawa, 1982) or MTs (Hirokawa, 1982; Benshalom and Reese, 1985; Price et al., 1991).

\section{$\mathrm{NF}$ and NF-sidearm interactions with brain mitochondria}

Several studies provide evidence for binding of mitochondria to IFs (Mose-Larsen et al., 1982; Eckert, 1986; Dudani et al., 1990; Olson and Winfrey, 1990; Stromer and Bendayan, 1990; Leterrier et al., 1991, 1994; Almahbobi et al., 1993; Collier et al., 1993; Reipert et al., 1999). In some but not all cell types (Mose-Larsen et al., 1982), MT disruption with colchicine or vinblastine also 


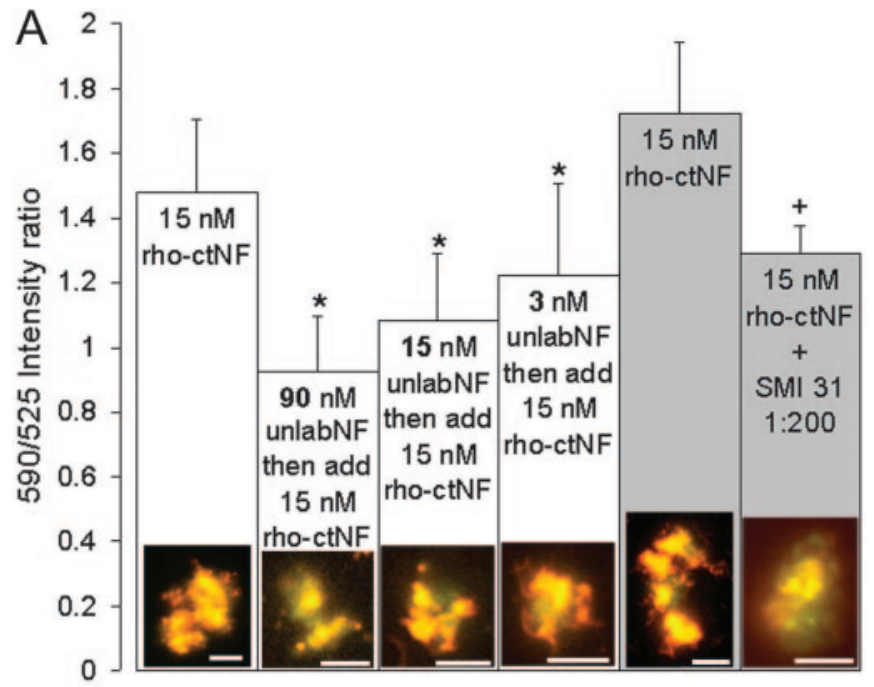

B

C
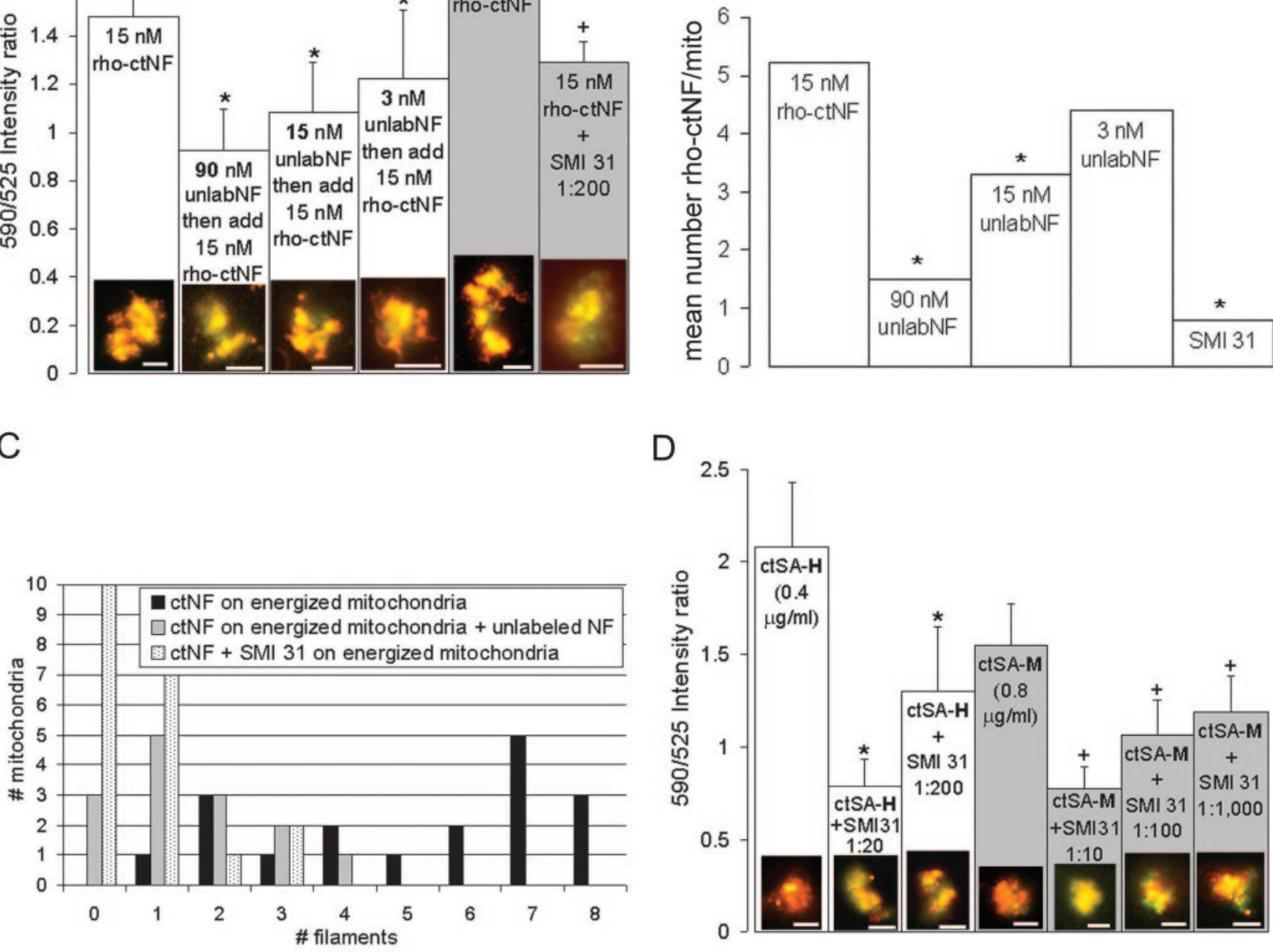

E

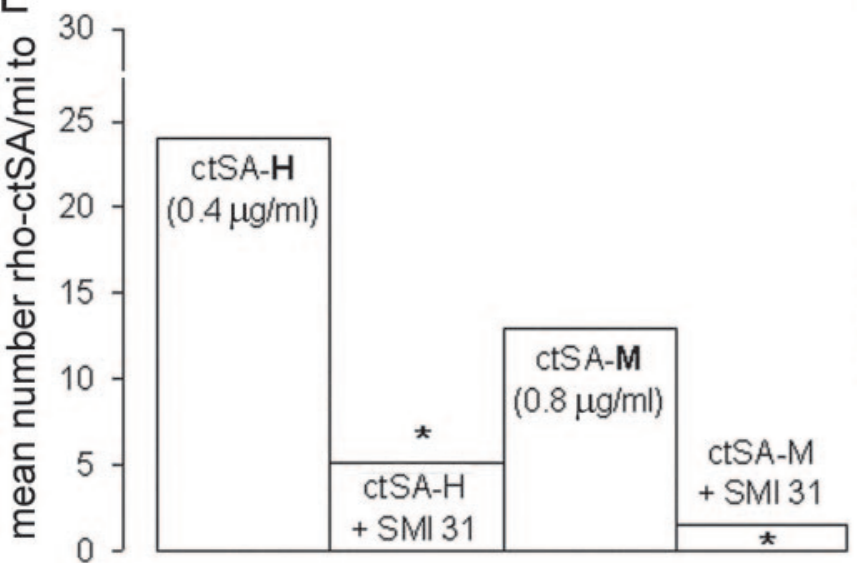

$\mathrm{F}$
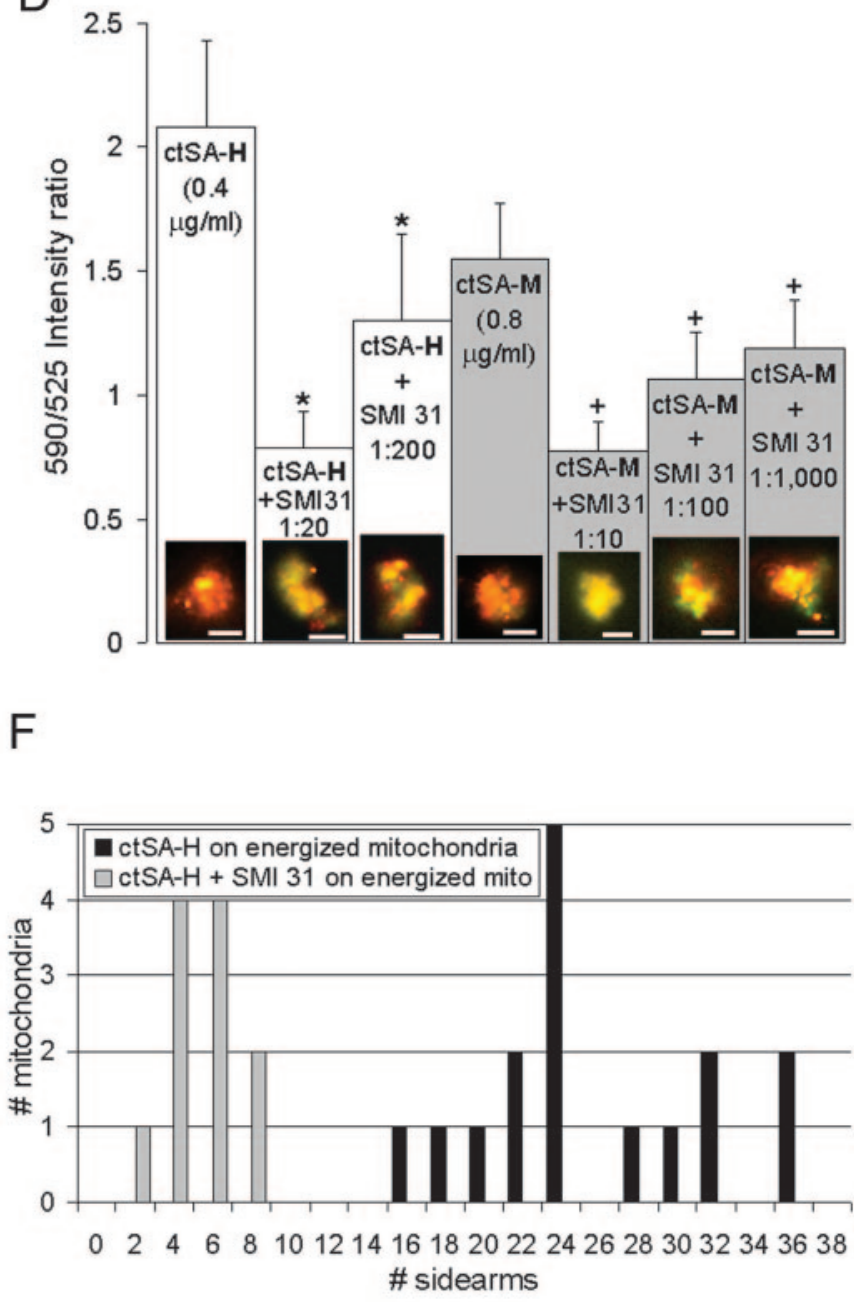

Figure 6. Specificity of mitochondria-NF and mitochondria-SA interactions. Specificity of mitochondria-NF/SA interactions was investigated by incubating mitochondria with unlabeled NFs before adding rho-NFs or by incubating NFs/SAs with monoclonal anti-NF antibodies before adding to mitochondria. $A, 0$ pen bars indicate $590 / 525 \mathrm{~nm}$ intensity ratio of energized mitochondria incubated without or with 90 , 15 , or $3 \mathrm{~nm}$ unlabeled NFs for $20 \mathrm{~min}$ before the addition of $15 \mathrm{~nm} \mathrm{rho-ctNFs.} \mathrm{Closed} \mathrm{bars} \mathrm{indicate} \mathrm{590/525} \mathrm{intensity} \mathrm{ratio} \mathrm{of} \mathrm{energized} \mathrm{mitochondria} \mathrm{mixed} \mathrm{with} \mathrm{ctNFs} \mathrm{preincubated} \mathrm{without} \mathrm{and} \mathrm{with} \mathrm{antibodies}$ against ctNFs (SMI 31). B, Mean numbers of rho-ctNFs attached per mitochondrion incubated without or with unlabeled ctNFs. Lowest values were obtained by the addition of rho-ctNFs preincubated (20 min) with SMI31 (1:200) to mitochondria (in the absence of unlabeled NFS). C, Distributions of filament counts as shown in $B$ (unlabeled NFs $=90 \mathrm{~nm}$ ). D, Intensity ratio (590/525) of energized mitochondria interacting with ctSA-Hs $(0.39 \mu \mathrm{g} / \mathrm{ml})$ (open bars) and ctSA-Ms $(0.8 \mu \mathrm{g} / \mathrm{ml})$ (closed bars) in the presence or absence of SMI 31. The binding of ctSA-Ms is statistically lower ( $p<0.01)$ compared with that of ctSA-Hs (compare first and fourth bar). E, Mean numbers of rho-ctSA-Hs and -Ms, in the presence or absence of SMI 31, attached per mitochondrion (SMI dilutions were 1:200 for SA-Hs and 1:100 for SA-Ms). F, Distributions of rho-SA-Hs $(0.1 \mu \mathrm{g} / \mathrm{ml})$ counts as shown in $E$. Scale bar of representative mitochondria for each condition $=1 \mu \mathrm{m}$. 

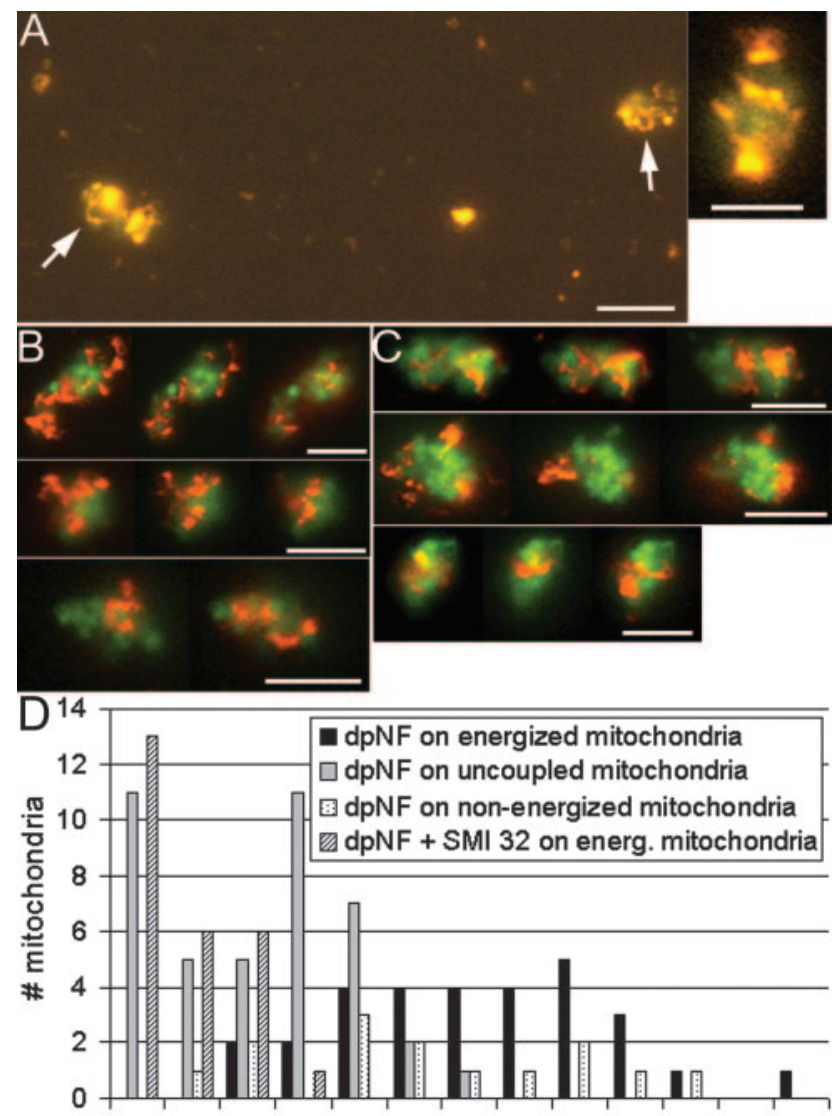

$\begin{array}{lllllllllllll}0 & 1 & 2 & 3 & 4 & 5 & 6 & 7 & 8 & 9 & 10 & 11 & 12\end{array}$

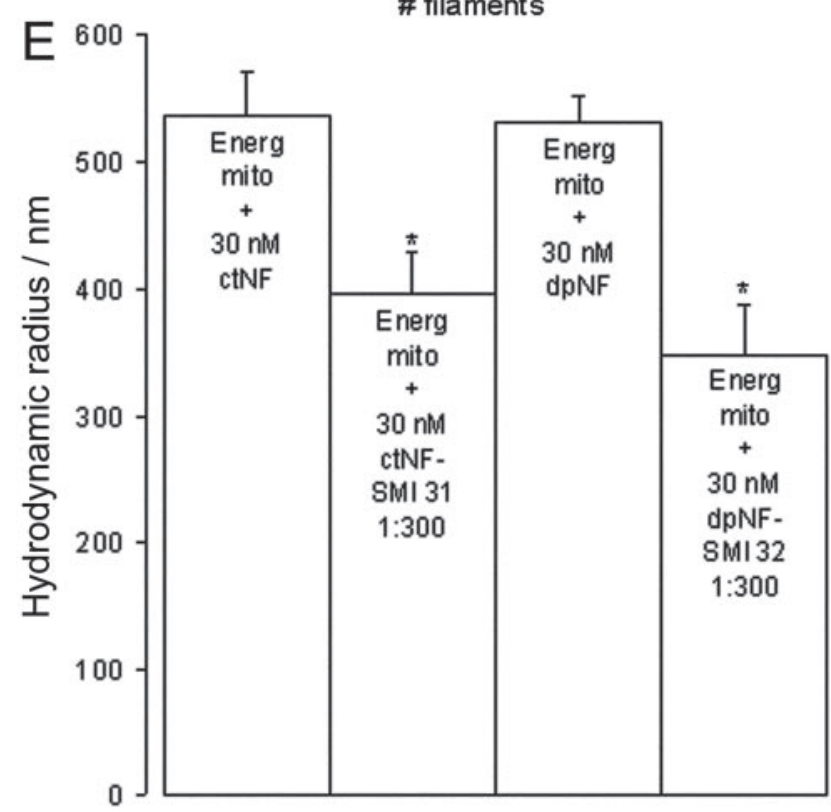

Figure 7. Effect of dephosphorylation on the binding of NFs to mitochondria. A, Binding of rhodamine-labeled dpNFs (pseudocolored red) to energized mitochondria (arrows point to single dpNFs) is lower compared with that of ctNFs (compare Fig. 3A, Table 1); however, dpNFs interact also with nonenergized $(B)$ or uncoupled ( $C$ mitochondria (each row of images in $B$ and $($ show, from left to right, different Z-sections). D, Quantification of dpNFs bound to energized (closed bars), nonenergized (dotted bars), and uncoupled (gray bars) mitochondria. In the presence of the anti-NF antibody SMI 32 (1:500), binding to energized mitochondria was significantly reduced. E, Specificity of binding of unlabeled ctNFs and dpNFs to unstained energized mitochondria $(0.1 \mathrm{mg} / \mathrm{ml})$ in equilibrium as revealed by DLS. The monoclonal antibody prevents binding of both ctNFs and dpNFs to the mitochondrion, which is reflected by a significant reduction of the hydrodynamic radius. Scale bar of representative mitochondria for each condition $=1 \mu \mathrm{m}$.
Table 2. Mean numbers of dephosphorylated filaments (dpNFs) and sidearms (dpSAs) binding per mitochondrion (data from Figs. 7D, 8)

\begin{tabular}{lll}
\hline & dpNFs & dpSAs \\
\hline Energized & $6.3 \mathrm{NF} /$ mito & $2.7 \mathrm{SA} /$ mito \\
Nonenergized & $5.4 \mathrm{NF} /$ mito (n.s.) & $3.3 \mathrm{SA} /$ mito (n.s.) \\
FCCP & $2.2 \mathrm{NF} /$ mito $(p<0.01)$ & $1.8 \mathrm{SA} /$ mito (n.s.) \\
\hline
\end{tabular}

Statistics were analyzed using Student's $t$ test on the basis of the comparison of energized states with the nonenergized or uncoupled states. The difference between ctNFs/energized (Table 1) and dpNFs/energized (Table 2) is not significant (n.s.) (data not shown). mito, Mitochondrion.

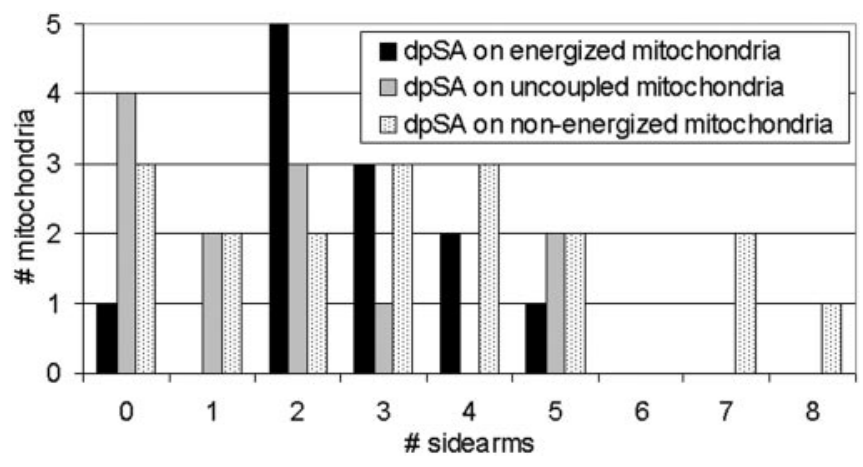

Figure 8. Binding of dephosphorylated sidearms to mitochondria. Quantification of rho$\mathrm{dpSA}-\mathrm{H}(0.1 \mu \mathrm{g} / \mathrm{ml})$ binding to mitochondria (revealed from fluorescence images; data not shown) shows a similar binding pattern as found for dpNFs (compare Fig. 7D, Table 2).

causes the disruption of IFs that correlates with the redistribution of mitochondria (Summerhayes et al., 1983). Disrupting IFs by acrylamide in Potorous tridactylis kidney 1 cells leads to a dramatic change in the organization of cytoplasmic organelles (Eckert, 1986). According to Martz et al. (1984), stationary axonal mitochondria vary in their proximity to MTs, and motile mitochondria are nearly always found at significant distances from either F-actin or IFs. This observation is consistent with those of Schnapp and Reese (1982), who suggested that mitochondria attached to filamentous elements other than MTs are probably stationary. The observations by Straube-West et al. (1996), who induced the selective retention of mitochondria in the cell body of cultured neurons by microinjection of an excess of NF-Hs, suggest that regulated NF-H-mediated binding of mitochondria to NFs is required for normal translocation of mitochondria in axons, likely by an alternation of motion along MTs and transient immobilization on NFs. Similar conclusions were reached in a recent report studying the perinuclear aggregation of mitochondria induced by the overexpression of NF-Hs in cultured cells (Szebenyi et al., 2002). In light of these data, demonstrating that an excess of free NF-H molecules affects the distribution of mitochondria in living cells, it would be of major interest to study the saltatory motion of mitochondria in cultivated neurons of NF-H tail-less mutant mice (Rao et al., 2002) according to the procedures used by Overly et al. (1996). No indications of abnormal mitochondria distribution in vivo were reported by Rao et al. (2002) in the neurons of NF-H tail-less mice; however, quantitative differences in such features as bidirectional motility or pausing time of mitochondria in cells may not be detected without real-time measurements of mitochondrial motility. Selective entrapment by NFs as suggested by our in vitro studies is not necessarily the only localization mechanism in vivo, and compensatory effects in the neurons of living organism are likely. Evidence provided in the present study that SA-Ms may also mediate interactions between NFs and mitochondria (Fig. 6D,E) suggests that potential alterations of mitochondrial motility in neurons 
from NF-H tail-less mice could be compensated by the contribution of SA-Ms to the local immobilization of the organelle, similar to the compensatory involvement of SA-Ms in other aspects of the axonal structure and function, as discussed by Rao et al. (2002).

In the present study, we confirm by direct observations that NFs bind to brain mitochondria and that this binding is mediated by the NF-H and NF-M sidearms. This finding is in agreement with previous studies showing that purified NF-Hs and NF-Ms compete with MAP2 for binding to brain mitochondria in vitro (Leterrier et al., 1990, 1991). The binding sites for MAP2 include the voltage-dependent anion channel (porin) (Leterrier et al., 1994; Linden and Karlsson, 1996), and several of the MAP2 binding proteins in mitochondrial membranes also bind the isolated NF-M subunit (Leterrier et al., 1994). It is thus possible that NF and SA binding to mitochondria are mediated by porin molecules or other cytoskeleton-binding proteins of the outer membrane localized in the porin-rich domains containing most of the binding sites for MAP2 (Leterrier et al., 1994). The present finding, that the binding of NFs to mitochondria is strongly affected by the potential of the mitochondrial membranes, suggests that the conformation of NF-binding proteins or the organization of the porin-rich domains is modified by the membrane potential of the organelle.

The possibility that NFs bind to mitochondrial subdomains enriched in a putative receptor is on the basis of the finding that MAPs bind as clusters on the mitochondrial surface, similar to clusters of porin (Leterrier et al., 1994). Together with recent data demonstrating the colocalization of porin and bound MAPs molecules in such restricted membrane domains (J.-F. Leterrier and M. Linden, unpublished data), several observations of the present work showing bound NF or SA aggregates on mitochondrial surface are in favor of such a hypothesis (Figs. $3 A, D, 5 A-D, 6 A, D$, $7 A-C)$. Furthermore, the finding that bound NFs are crosslinked to the membrane surface by regular cross-bridges suggests that several receptors for the same filament are present on the surface of mitochondria (Fig. $3 H$ ).

Our finding that NFs tend to aggregate on the mitochondrial surface (Figs. $3 A, D, E 1,6 A, 7$ ) is in contrast to the study of Kumar et al. (2002), which claims that NFs do not interact but instead are apart within distances imposed by repulsion between charged sidearms. In contrast, other reports demonstrate the occurrence of cross-bridges between NFs in situ (Hirokawa, 1982; Hirokawa et al., 1984) and the occurrence of interconnected bundles that can be isolated as such from the tissue (Shaw and Hou, 1990; Yabe et al., 2001). From these differing findings, NF interactions in vivo are likely to involve a highly controlled equilibrium between single and interconnected NFs. Previous studies of native NFs demonstrated their bundling in vitro into semiparallel arrays of filaments similar to axonal bundles, mediated by bivalent cations. This mechanism is regulated by NF phosphorylation and several NF-associated proteins and enzymes (Leterrier et al., 1996; Gou et al., 1998), thus suggesting that closely related biochemical events may occur in vivo. The occurrence of interactions between NFs in the present experiments is likely a property of native NFs instead of being the consequence of protein denaturation.

\section{Mitochondrial-NF associations mediated by membrane potential}

The mitochondrial membrane potential drives synthesis of ATP, calcium accumulation, and maintenance of ion gradients that permit the influx of substrates and efflux of metabolic products. Changes in membrane potential may change mitochondrial dis- tribution in neurons regarding areas of high energy demand, e.g., Ranvier nodes, dendrites, growth cones, and synapses (Peters et al., 1991; Fabricius et al., 1993).

It has been shown by Overly et al. (1996), using JC-1 with cultured neurons, that differences in mitochondrial membrane potential exist between axons and dendrites: a higher ratio of charged/uncharged mitochondria was found in dendrites as compared with axons, and twice as many mitochondria were motile in axons as in dendrites. Because phosphorylated NFs are much more abundant in axons than in dendrites (Carden et al., 1987; Foster et al., 1987; Brown, 1998), these observations may be consistent with the present study showing that ctNFs do not associate strongly in vitro with low-energized mitochondria. Evidence that nonenergized (non-FCCP treated) mitochondria do not bind to NFs in vitro may result in vivo in the abolition of the pausing of the organelle along NFs, allowing a more straightforward movement of mitochondria in axons. Differences in organelle transport between axons and dendrites, however, can also be on the basis of differences in the physiological states of the neurites (Overly et al., 1996; Ligon and Steward, 2000), the cytoskeletal composition and organization within the two domains (Baas et al., 1988; Sheetz and Martenson, 1991), and the phosphorylation level of motor proteins (Hamasaki et al., 1991; McIlvain et al., 1994; Lee and Hollenbeck, 1995; Morfini et al., 2002).

\section{Effect of NF phosphorylation on mitochondria-NF interactions}

Poorly phosphorylated NFs dominate in cell bodies, dendrites, and the proximal part of the axon (Carden et al., 1987; Foster et al., 1987; Brown, 1998), their projections are shorter and more compact (Gou et al., 1998), and they form more rarely (irregular) inter-NF cross-bridges than phosphorylated NFs (Gotow et al., 1994). The present study shows that extensive dephosphorylation of NFs affects their interactions with mitochondria in a subtle manner. Both ctNFs and dpNFs bind strongly to energized mitochondria. Although the binding of ctNFs is depends on a high potential of the organelle, and bound ctNFs are released from mitochondria when membrane potential is lowered (Fig. 3D, Table 1), dpNFs bind mitochondria of low and high potential with a similar efficiency (Table 2). Uncoupling of mitochondria by FCCP reduces the overall binding of both phosphorylated and dephosphorylated NFs or SAs (Tables 1,2), suggesting that phosphorylation may regulate the binding affinity of NFs for mitochondria in a potential-dependent manner.

Thus, these findings raise the possibility that the heterogeneous phosphorylation pattern of NFs (Brown, 1998) determines the local strength of mitochondria-NF interactions in concert with the energy status of mitochondria, thereby controlling mitochondrial motility through the length of their pausing time. The larger number of motile mitochondria exhibiting long uninterrupted excursion found in axons compared with dendrites (Overly et al., 1996; Ligon and Steward, 2000) may involve such mechanisms. Evidence that NF phosphorylation is correlated with a selective binding to energized mitochondria may be significant with regard to the aberrant distribution of mitochondria in aging and neurodegenerative diseases (e.g., amyotrophic lateral sclerosis, Alzheimer's disease), in which the aggregation of hyperphosphorylated NFs is a common hallmark (for review, see Leung et al., 1998; Shaw, 1998).

\section{Conclusion}

A variety of biological evidence suggests that mitochondria can be immobilized on the cytoskeleton in a regulated manner and that IFs are an important structure for this process. The factors and 
conditions regulating these interactions are unknown and difficult to discern from whole-cell measurements. The in vitro approach taken here shows directly that the metabolic status of mitochondria and the phosphorylation level of NFs can control the immobilization of mitochondria on NF networks and thereby suggests these effects as factors directing mitochondrial localization in vivo.

\section{References}

Almahbobi G, Williams LJ, Han XG, Hall PF (1993) Binding of lipid droplets and mitochondria to intermediate filaments in rat Leydig cells. J Reprod Fertil 98:209-217.

Aranda-Espinoza H, Carl P, Leterrier JF, Janmey P, Discher DE (2002) Domain unfolding in neurofilament sidearms: effects of phosphorylation and ATP. FEBS Lett 531:397-401.

Aw TY, Jones DP (1988) Microzonation of ATP and $\mathrm{pH}$ in the aqueous cytoplasm of mammalian cells. In: Microcompartmentation, pp 191-208. Boca Raton, FL: CRC.

Baas PW, Deitch JS, Black MM, Banker GA (1988) Polarity orientation of microtubules in hippocampal neurons: uniformity in the axon and nonuniformity in the dendrite. Proc Natl Acad Sci USA 85:8335-8339.

Benshalom G, Reese TS (1985) Ultrastructural observations on the cytoarchitecture of axons processed by rapid-freezing and freeze-substitution. J Neurocytol 14:943-960.

Bereiter-Hahn J, Vöth M (1994) Dynamics of mitochondria in living cells: shape changes, dislocations, fusion, and fission of mitochondria. Microsc Res Tech 27:198-219.

Brown A (1998) Contiguous phosphorylated and non-phosphorylated domains along axonal neurofilaments. J Cell Sci 111:455-467.

Carden MJ, Schlaepfer WW, Lee VM (1985) The structure, biochemical properties, and immunogenicity of neurofilament peripheral regions are determined by phosphorylation state. J Biol Chem 260:9805-9817.

Carden MJ, Trojanowski JQ, Schlaepfer WW, Lee VM (1987) Two-stage expression of neurofilament polypeptides during rat neurogenesis with early establishment of adult phosphorylation patterns. J Neurosci 7:3489-3504.

Chin TK, Harding SE, Eagles PA (1989) Characterization of two proteolytically derived soluble polypeptides from the neurofilament triplet components NFM and NFH. Biochem J 264:53-60.

Collier NC, Sheetz MP, Schlesinger MJ (1993) Concomitant changes in mitochondria and intermediate filaments during heat shock and recovery of chicken embryo fibroblasts. J Cell Biochem 52:297-307.

Couchman JR, Rees DA (1982) Organelle-cytoskeleton relationships in fibroblasts: mitochondria, Golgi apparatus, and endoplasmic reticulum in phases of movement and growth. Eur J Cell Biol 27:47-54.

Dudani AK, Austin RC, Venner TJ, Gupta RS (1990) Effects of antimitotic and antimitochondrial agents on the cellular distribution of microtubules and mitochondria. Cytobios 63:95-108.

Eckert BS (1986) Alteration of the distribution of intermediate filaments in PtK1 cells by acrylamide. II: Effect on the organization of cytoplasmic organelles. Cell Motil Cytoskeleton 6:15-24.

Eyer J, Leterrier JF (1988) Influence of the phosphorylation state of neurofilament proteins on the interactions between purified filaments in vitro. Biochem J 252:655-660.

Fabricius C, Berthold CH, Rydmark M (1993) Axoplasmic organelles at nodes of Ranvier. II. Occurrence and distribution in large myelinated spinal cord axons of the adult cat. J Neurocytol 22:941-954.

Fawcett DW (1981) Mitochondria. In: The cell, pp 410-484. Philadelphia: W. B. Saunders

Forman DS, Lynch KJ, Smith RS (1987) Organelle dynamics in lobster axons: anterograde, retrograde and stationary mitochondria. Brain Res 412:96-106.

Foster GA, Dahl D, Lee VM (1987) Temporal and topographic relationships between the phosphorylated and nonphosphorylated epitopes of the 200 $\mathrm{kDa}$ neurofilament protein during development in vitro. J Neurosci 7:2651-2663.

Glatter O, Sieberer J, Schnablegger H (1991) A comparative study on different scattering techniques and data evaluation methods for sizing of colloidal systems using light scattering. Part Part Syst Charact 8:274-281.

Gotow T, Tanaka T, Nakamura Y, Takeda M (1994) Dephosphorylation of the largest neurofilament subunit protein influences the structure of cross-bridges in reassembled neurofilaments. J Cell Sci 107:1949-1957.
Gou JP, Gotow T, Janmey PA, Leterrier JF (1998) Regulation of neurofilament interactions in vitro by natural and synthetic polypeptides sharing Lys-Ser-Pro sequences with the heavy neurofilament subunit NF-H: neurofilament cross-bridging by antiparallel sidearm overlapping. Med Biol Eng Comput 36:371-387.

Hackenbrock CR (1966) Ultrastructural bases for metabolically linked mechanical activity in mitochondria. I. Reversible ultrastructural changes with change in metabolic steady state in isolated liver mitochondria. J Cell Biol 30:269-297.

Halestrap AP (1989) The regulation of the matrix volume of mammalian mitochondria in vivo and in vitro and its role in the control of mitochondrial metabolism. Biochim Biophys Acta 973:355-382.

Hamasaki T, Barkalow K, Richmond J, Satir P (1991) cAMP-stimulated phosphorylation of an axonemal polypeptide that copurifies with the $22 \mathrm{~S}$ dynein arm regulates microtubule translocation velocity and swimming speed in Paramecium. Proc Natl Acad Sci USA 88:7918-7922.

Hirokawa N (1982) Cross-linker system between neurofilaments, microtubules, and membranous organelles in frog axons revealed by the quickfreeze, deep-etching method. J Cell Biol 94:129-142.

Hirokawa N, Glicksman MA, Willard MB (1984) Organization of mammalian neurofilament polypeptides within the neuronal cytoskeleton. J Cell Biol 98:1523-1536.

Hisanaga S, Yasugawa S, Yamakawa T, Miyamoto E, Ikebe M, Uchiyama M, Kishimoto T (1993) Dephosphorylation of microtubule-binding sites at the neurofilament- $\mathrm{H}$ tail domain by alkaline, acid, and protein phosphatases. J Biochem (Tokyo) 113:705-709.

Hollenbeck PJ (1996) The pattern and mechanism of mitochondrial transport in axons. Front Biosci 1:d91-d102.

Julien JP, Mushynski WE (1982) Multiple phosphorylation sites in mammalian neurofilament polypeptides. J Biol Chem 257:10467-10470.

Julien JP, Mushynski WE (1983) The distribution of phosphorylation sites among identified proteolytic fragments of mammalian neurofilaments. J Biol Chem 258:4019-4025.

Kas J, Strey H, Tang JX, Finger D, Ezzell R, Sackmann E, Janmey PA (1996) F-actin, a model polymer for semiflexible chains in dilute, semidilute, and liquid crystalline solutions. Biophys J 70:609-625.

Kellermayer M, Ludany A, Jobst K, Szucs G, Trombitas K, Hazlewood CF (1986) Cocompartmentation of proteins and $\mathrm{K}^{+}$within the living cell. Proc Natl Acad Sci USA 83:1011-1015.

Krendel M, Sgourdas G, Bonder EM (1998) Disassembly of actin filaments leads to increased rate and frequency of mitochondrial movement along microtubules. Cell Motil Cytoskeleton 40:368-378.

Kristal BS, Dubinsky JM (1997) Mitochondrial permeability transition in the central nervous system: induction by calcium cycling-dependent and -independent pathways. J Neurochem 69:524-538.

Kumar S, Yin X, Trapp BD, Paulaitis ME, Hoh JH (2002) Role of long-range repulsive forces in organizing axonal neurofilament distributions: evidence from mice deficient in myelin-associated glycoprotein. J Neurosci Res 68:681-690.

Laemmli UK (1970) Cleavage of structural proteins during the assembly of the head of bacteriophage T4. Nature 227:680-685.

Lazzarino DA, Boldogh I, Smith MG, Rosand J, Pon LA (1994) Yeast mitochondria contain ATP-sensitive, reversible actin-binding activity. Mol Biol Cell 5:807-818.

Lee KD, Hollenbeck PJ (1995) Phosphorylation of kinesin in vivo correlates with organelle association and neurite outgrowth. J Biol Chem 270:5600-5605.

Leterrier JF, Linden M, Nelson BD (1990) How do microtubules interact in vitro with purified subcellular organelles? Biochem J 269:556-558.

Leterrier JF, Weiss DG, Linden M (1991) In vitro studies of the physical interactions between neurofilaments, microtubules and mitochondria isolated from the central nervous system. Am Inst Phys Conf Proc 226:91-105.

Leterrier JF, Rusakov DA, Nelson BD, Linden M (1994) Interactions between brain mitochondria and cytoskeleton: evidence for specialized outer membrane domains involved in the association of cytoskeletonassociated proteins to mitochondria in situ and in vitro. Microsc Res Tech 27:233-261.

Leterrier JF, Kas J, Hartwig J, Vegners R, Janmey PA (1996) Mechanical effects of neurofilament cross-bridges. Modulation by phosphorylation, lipids, and interactions with F-actin. J Biol Chem 271:15687-15694. 
Leung CL, Flores RL, Liem RK (1998) The complexity of intermediate filaments in the nervous system. Subcell Biochem 31:497-526.

Lifshitz J, Friberg H, Neumar RW, Raghupathi R, Welsh FA, Janmey P, Saatman KE, Wieloch T, Grady MS, McIntosh TK (2003) Structural and functional damage sustained by mitochondria after traumatic brain injury in the rat: evidence for differentially sensitive populations in the cortex and hippocampus. J Cereb Blood Flow Metab 23:219-231.

Ligon LA, Steward O (2000) Movement of mitochondria in the axons and dendrites of cultured hippocampal neurons. J Comp Neurol 427:340-350.

Linden M, Karlsson G (1996) Identification of porin as a binding site for MAP2. Biochem Biophys Res Commun 218:833-836.

Linden M, Li Z, Paulin D, Gotow T, Leterrier JF (2001) Effects of desmin gene knockout on mice heart mitochondria. J Bioenerg Biomembr 33:333-341.

Lowry OH, Rosebrough NJ, Farr AL, Randall RJ (1951) Protein measurement using the Folin phenol reagent. J Biol Chem 193:265-275.

Martz D, Lasek RJ, Brady ST, Allen RD (1984) Mitochondrial motility in axons: membranous organelles may interact with the force generating system through multiple surface binding sites. Cell Motil 4:89-101.

McIlvain Jr JM, Burkhardt JK, Hamm-Alvarez S, Argon Y, Sheetz MP (1994) Regulation of kinesin activity by phosphorylation of kinesin-associated proteins. J Biol Chem 269:19176-19182.

Milner DJ, Mavroidis M, Weisleder N, Capetanaki Y (2000) Desmin cytoskeleton linked to muscle mitochondrial distribution and respiratory function. J Cell Biol 150:1283-1298.

Morfini G, Szebenyi G, Elluru R, Ratner N, Brady ST (2002) Glycogen synthase kinase 3 phosphorylates kinesin light chains and negatively regulates kinesin-based motility. EMBO J 21:281-293.

Morris RL, Hollenbeck PJ (1993) The regulation of bidirectional mitochondrial transport is coordinated with axonal outgrowth. J Cell Sci 104:917-927.

Morris RL, Hollenbeck PJ (1995) Axonal transport of mitochondria along microtubules and F-actin in living vertebrate neurons. J Cell Biol 131:1315-1326.

Mose-Larsen P, Bravo R, Fey SJ, Small JV, Celis JE (1982) Putative association of mitochondria with a subpopulation of intermediate-sized filaments in cultured human skin fibroblasts. Cell 31:681-692.

Olson GE, Winfrey VP (1990) Mitochondria-cytoskeleton interactions in the sperm midpiece. J Struct Biol 103:13-22.

Overly CC, Rieff HI, Hollenbeck PJ (1996) Organelle motility and metabolism in axons vs dendrites of cultured hippocampal neurons. J Cell Sci 109:971-980.

Peters A, Palay SL, Webster HD (1991) The fine structure of the nervous system: the neurons and supporting cells. New York: Oxford UP.

Price RL, Lasek RJ, Katz MJ (1991） Microtubules have special physical associations with smooth endoplasmic reticula and mitochondria in axons. Brain Res 540:209-216.

Rao MV, Garcia ML, Miyazaki Y, Gotow T, Yuan A, Mattina S, Ward CM, Calcutt NA, Uchiyama Y, Nixon RA, Cleveland DW (2002) Gene replacement in mice reveals that the heavily phosphorylated tail of neurofilament heavy subunit does not affect axonal caliber or the transit of cargoes in slow axonal transport. J Cell Biol 158:681-693.

Reers M, Smith TW, Chen LB (1991) J-aggregate formation of a carbocyanine as a quantitative fluorescent indicator of membrane potential. Biochemistry 30:4480-4486.

Reers M, Smiley ST, Mottola-Hartshorn C, Chen A, Lin M, Chen LB (1995)
Mitochondrial membrane potential monitored by JC-1 dye. Methods Enzymol 260:406-417.

Reipert S, Steinbock F, Fischer I, Bittner RE, Zeold A, Wiche G (1999) Association of mitochondria with plectin and desmin intermediate filaments in striated muscle. Exp Cell Res 252:479-491.

Salvioli S, Ardizzoni A, Franceschi C, Cossarizza A (1997) JC-1, but not DiOC6(3) or rhodamine 123, is a reliable fluorescent probe to assess delta psi changes in intact cells: implications for studies on mitochondrial functionality during apoptosis. FEBS Lett 411:77-82.

Schnapp BJ, Reese TS (1982) Cytoplasmic structure in rapid-frozen axons. J Cell Biol 94:667-669.

Shah JV, Flanagan LA, Janmey PA, Leterrier JF (2000) Bidirectional translocation of neurofilaments along microtubules mediated in part by dynein/dynactin. Mol Biol Cell 11:3495-3508.

Shaw G (1998) Neurofilament involvement in disease processes. In: Neurofilaments, pp 243-263. New York: Springer.

Shaw G, Hou ZC (1990) Bundling and cross-linking of intermediate filaments of the nervous system. J Neurosci Res 25:561-568.

Sheetz MP (1999) Motor and cargo interactions. Eur J Biochem 262:19-25.

Sheetz MP, Martenson CH (1991) Axonal transport: beyond kinesin and cytoplasmic dynein. Curr Opin Neurobiol 1:393-398.

Sims NR (1990) Rapid isolation of metabolically active mitochondria from rat brain and subregions using Percoll density gradient centrifugation. J Neurochem 55:698-707.

Smiley ST, Reers M, Mottola-Hartshorn C, Lin M, Chen A, Smith TW, Steele Jr GD, Chen LB (1991) Intracellular heterogeneity in mitochondrial membrane potentials revealed by a J-aggregate-forming lipophilic cation JC-1. Proc Natl Acad Sci USA 88:3671-3675.

Smith MG, Simon VR, O’Sullivan H, Pon LA (1995) Organelle-cytoskeletal interactions: actin mutations inhibit meiosis-dependent mitochondrial rearrangement in the budding yeast Saccharomyces cerevisiae. Mol Biol Cell 6:1381-1396.

Straube-West K, Loomis PA, Opal P, Goldman RD (1996) Alterations in neural intermediate filament organization: functional implications and the induction of pathological changes related to motor neuron disease. J Cell Sci 109:2319-2329.

Stromer MH, Bendayan M (1990) Immunocytochemical identification of cytoskeletal linkages to smooth muscle cell nuclei and mitochondria. Cell Motil Cytoskeleton 17:11-18.

Summerhayes IC, Wong D, Chen LB (1983) Effect of microtubules and intermediate filaments on mitochondrial distribution. J Cell Sci 61:87-105.

Szebenyi G, Smith GM, Li P, Brady ST (2002) Overexpression of neurofilament $\mathrm{H}$ disrupts normal cell structure and function. J Neurosci Res 68:185-198.

Toh BH, Lolait SJ, Mathy JP, Baum R (1980) Association of mitochondria with intermediate filaments and of polyribosomes with cytoplasmic actin. Cell Tissue Res 211:163-169.

Towbin H, Staehelin T, Gordon J (1979) Electrophoretic transfer of proteins from polyacrylamide gels to nitrocellulose sheets: procedure and some applications. Proc Natl Acad Sci USA 76:4350-4354.

Wong-Riley MT (1989) Cytochrome oxidase: an endogenous metabolic marker for neuronal activity. Trends Neurosci 12:94-101.

Yabe JT, Chylinski T, Wang FS, Pimenta A, Kattar SD, Linsley MD, Chan WK, Shea TB (2001) Neurofilaments consist of distinct populations that can be distinguished by C-terminal phosphorylation, bundling, and axonal transport rate in growing axonal neurites. J Neurosci 21:2195-2205. 